The Astrophysical Journal, 647:444-463, 2006 August 10

(C) 2006. The American Astronomical Society. All rights reserved. Printed in U.S.A.

\title{
FEW SKEWED DISKS FOUND IN FIRST CLOSURE-PHASE SURVEY OF HERBIG Ae/Be STARS
}

\author{
J. D. Monnier, ${ }^{1}$ J.-P. Berger,${ }^{2}$ R. Millan-Gabet ${ }^{3}$ W. A. Traub ${ }^{4,5}$ F. P. Schloerb,${ }^{6}$ E. Pedretti, ${ }^{1}$ M. Benisty, ${ }^{2}$ \\ N. P. Carleton, ${ }^{4}$ P. Haguenauer ${ }^{7}$ P. Kern, ${ }^{2}$ P. Labeye, ${ }^{8}$ M. G. Lacasse, ${ }^{4}$ F. Malbet ${ }^{2}$ \\ K. Perraut, ${ }^{2}$ M. Pearlman, ${ }^{4}$ and M. Zhao ${ }^{1}$ \\ Received 2006 February 2; accepted 2006 April 17
}

\begin{abstract}
Using the three-telescope IOTA interferometer on Mount Hopkins, we report results from the first near-infrared $(\lambda=1.65 \mu \mathrm{m})$ closure-phase survey of young stellar objects (YSOs). These closure phases allow us to unambiguously detect departures from centrosymmetry (i.e., skew) in the emission pattern from YSO disks on the scale of 4 mas, expected from generic "flared disk" models. Six of 14 targets showed small, yet statistically significant nonzero closure phases, with largest values from the young binary system MWC 361-A and the (pre-main-sequence?) Be star HD 45677. Our observations are quite sensitive to the vertical structure of the inner disk, and we confront the predictions of the "puffed-up inner wall" models of Dullemond, Dominik, \& Natta (DDN). Our data support disk models with curved inner rims because the expected emission appears symmetrically distributed around the star over a wide range of inclination angles. In contrast, our results are incompatible with the models possessing vertical inner walls because they predict extreme skewness (i.e., large closure phases) from the near-IR disk emission that is not seen in our data. In addition, we also present the discovery of mysterious $H$-band "halos" ( $\sim 5 \%-10 \%$ of light on scales $0.01-0.50$ ) around a few objects, a preliminary "parametric imaging" study for HD 45677, and the first astrometric orbit for the young binary MWC 361-A.

Subject headings: accretion, accretion disks - binaries: spectroscopic - instrumentation: interferometers stars: individual (AB Aur, HD 45677, HD 144432, MWC 166, MWC 275, MWC 297, MWC 342, MWC 361, MWC 480, MWC 614, MWC 863, MWC 1080, RY Tau, v1295 Aql) stars: pre-main-sequence — techniques: interferometric
\end{abstract}

\section{INTRODUCTION}

The study of accretion disk evolution in young stellar objects (YSOs) promises to directly link the fields of star formation with planet formation. Spatially resolved, multiwavelength observations can directly probe the dust and gas distribution on solar system scales, allowing precise testing of disk models while simultaneously providing the crucial physical parameters for planet formation initial conditions. Even young Jupiter-mass planets embedded in natal disks should be detectable in the future.

Already, observations from long-baseline interferometers (IOTA, PTI, Keck) have empirically determined the near-infrared (NIR) emission sizes of YSOs (e.g., Millan-Gabet et al. 1999, 2001; Akeson et al. 2000; Eisner et al. 2003), finding the sizes to be strongly linked to the luminosity of the central stars (Monnier \& Millan-Gabet 2002; Monnier et al. 2005). These results are qualitatively explained by a model in which the NIR emission arises entirely from the hottest dust at the inner edge of an accretion disk, and the measured sizes correspond to the dustdestruction radius around the young stars (Tuthill et al. 2001; Natta et al. 2001). Recently, Eisner et al. (2004) reported con-

\footnotetext{
1 Astronomy Department, University of Michigan, 941 Dennison Building, Ann Arbor, MI 48109-1090; monnier@umich.edu.

${ }^{2}$ Laboratoire d'Astrophysique de Grenoble, 414 Rue de la Piscine 38400 Saint Martin d'Heres, France.

3 Michelson Science Center, California Institute of Technology, Pasadena, CA.

${ }^{4}$ Harvard-Smithsonian Center for Astrophysics, 60 Garden Street, Cambridge, MA 02138.

5 Jet Propulsion Laboratory, California Institute of Technology, Pasadena, CA.

6 University of Massachusetts, Amherst, MA.

Alcatel Space Industries, Cannes, France.

8 LETI-CEA, Grenoble, France.
}

vincing signs that NIR dust emission from many intermediatemass Herbig Ae/Be stars is elongated, implicating disklike structure as expected from star and planet formation theory.

The simple detection of "elongation" by an interferometer, however, does not well constrain the geometry of the emission region. For instance, current data cannot distinguish between (1) a centrosymmetric emission pattern as expected from a perfectly flat, inclined disk, (2) elongated emission arising preferentially from one side of the disk (as expected from optical depth effects associated with flaring; Malbet et al. 2001), or (3) hybrid disk+halo models with multiple emission components (Vinković et al. 2006). All current results are based on measuring interferometer fringe amplitudes, while interferometric fringe phase information is required to unambiguously detect deviations from simple symmetries. Ultimately, the phases and amplitudes are all needed to permit model-independent imaging using aperture synthesis techniques (Thompson et al. 2001).

While atmospheric turbulence corrupts the direct measurement of fringe phase, interferometrists using three or more telescopes can measure the "closure phase" (CP), a phase quantity that is unspoiled by telescope-specific phase errors (Jennison 1958; Monnier 2000). Recently, the Infrared Optical Telescope Array (IOTA) became the third facility to successfully achieve CP operation (Traub et al. 2003; Monnier et al. 2004) and is the only such facility that has demonstrated the capability to study YSOs (Millan-Gabet et al. 2006c).

Here we expand the original Herbig Ae/Be survey work of Millan-Gabet et al. (2001) using three IOTA telescopes simultaneously, tripling the rate at which visibility measurements are collected and allowing CPs to be measured for the first time for a sample of YSOs (see Millan-Gabet et al. [2006c] for the first results for the prototype Herbig Ae star AB Aur). This work has been made possible by the advanced beam combiner IONIC3 
TABLE 1

Basic Properties of Targets

\begin{tabular}{|c|c|c|c|c|c|c|c|c|}
\hline Target Names & $\begin{array}{c}\text { RA } \\
(\mathrm{J} 2000.0)\end{array}$ & $\begin{array}{c}\text { Decl. } \\
(\mathrm{J} 2000.0)\end{array}$ & $\begin{array}{c}V \\
\mathrm{Mag}^{\mathrm{a}}\end{array}$ & $\begin{array}{c}H \\
\mathrm{Mag}^{\mathrm{a}}\end{array}$ & Spectral Type ${ }^{\mathrm{b}}$ & $\begin{array}{l}\text { Distance }^{\mathrm{b}} \\
\quad(\mathrm{pc})\end{array}$ & $\begin{array}{l}\text { Adopted Luminosity } \\
\qquad\left(L_{\odot}\right)\end{array}$ & Photometry References \\
\hline RY Tau.. & 042157.40 & +282635.5 & 10.2 & 6.1 & F8 IIIe (1) & $142 \pm 14(2)$ & $5 \pm 2(3)$ & $4,5,6,7$ \\
\hline AB Aur . & 045941.53 & +40 5009.7 & 7.1 & 5.1 & A1 (11) & $144_{-17}^{+23}(12)$ & $70 \pm 20(3)$ & $4,5,6,7,9,10,13,14,15,16$ \\
\hline HD $45677^{\mathrm{c}}$, MWC $142 \ldots \ldots$. & 062817.42 & -130311.0 & 8.1 & 6.3 & B2 (17) & $000 \pm 500(17)$ & $14000 \pm 7000(3)$ & 4,18 \\
\hline MWC 166, H & 070425.52 & -102715.7 & 7.0 & 6.2 & B0 IVe (19) & $1150(20)$ & $100000 \pm 50$ & 4,5 , \\
\hline MWC 275, HD 163296......... & 175621.29 & -215721.8 & 6.9 & 5.5 & A1 Vepv (1) & $122_{-13}^{+17}(8,24)$ & $40 \pm 8(3)$ & $4,6,9,16,22$ \\
\hline MWC 297, NZ Ser ................. & 182739.53 & -034952.0 & 12.3 & 4.4 & B1 Ve (1) & $250(25)$ & $33000 \pm 13000(3)$ & $4,10,14,22$ \\
\hline MWC 614, HD 179218........ & 191111.24 & +154715.6 & 7.2 & 6.6 & A0 V (1) & $240_{-40}^{+70}(8,24)$ & $100 \pm 35(3)$ & $4,5,6,15,22,26$ \\
\hline v1295 Aql, HD $190073 . . . \ldots . .$. & 200302.51 & +054416.7 & 7.8 & 6.6 & A2 IVev (1) & $>290(8,24)$ & $>83(3)$ & $4,5,6,10$ \\
\hline MWC 342, v1972 Cyg.......... & 202303.61 & +392949.9 & 10.6 & 5.8 & B1 (27) & $1000(27)$ & $33000 \pm 15000(3)$ & $4,5,27,28,29$ \\
\hline MWC 361, HD 200775........ & 210136.91 & +68 0947.7 & 7.4 & 5.5 & B3 (11) + B? (30) & $430_{-90}^{+160}(8,24)$ & $6000 \pm 2000$ & $4,5,6,14$ \\
\hline
\end{tabular}

${ }^{\text {a }}$ Many of the targets are variable stars, and these magnitudes ( $V$ band from Simbad, and $H$ band from 2MASS) are merely representative.

${ }^{\mathrm{b}}$ Reference numbers for spectral type, distance and adopted luminosity follow in parentheses.

${ }^{c}$ Peculiar B[e] star with uncertain classification.

References.-(1) Mora et al. 2001; (2) Wichmann et al. 1998; (3) SED fitting, this work; (4) 2MASS, VizieR Online Data Catalog, 2246 (R. M. Cutri et al., 2003 ); (5) Tycho-2, Høg et al. 2000; (6) USNO-B Catalog, Monet et al. 2003; (7) Skrutskie et al. 1996; (8) van den Ancker et al. 1998; (9) Malfait et al. 1998; (10) Eisner et al. 2004; (11) Hernández et al. 2004; (12) Bertout et al. 1999; (13) Herbig \& Bell 1988; (14) Hillenbrand et al. 1992; (15) Guide Star Catalog 2.2, VizieR Online Data Catalog, 1271 (Space Telescope Science Institute, 2001); (16) VizieR Online Data Catalog, 2237 (J. R. Ducati, 2002); (17) de Winter \& van den Ancker 1997; (18) New MDM Observatory photometry from 2004 November, this work; (19) Finkenzeller 1985; (20) Herbst et al. 1982; (21) Pérez et al. 2004; (22) DENIS Database, VizieR Online Data Catalog, 2002 (C. D. Thé, 2005); (23) Sylvester et al. 1996; (24) VizieR Online Data Catalog, 1239 (ESA, 1997); (25) Drew et al. 1997; (26) Miroshnichenko et al. 1999b; (27) Miroshnichenko et al. 1999a; (28) Kharchenko 2001; (29) Bergner et al. 1995; (30) Pogodin et al. 2004; (31) Cohen \& Kuhi 1979; (32) Grankin et al. 1992.

(Berger et al. 2003), which exploits integrated optics technology developed for the telecommunication industry.

This article presents a large volume of data and analysis and is organized as follows. First, in $\S 2$ we describe the interferometer observations and present the data results in a summary form. In $\S 3$ we analyze the spectral energy distribution (SED) and visibility data together for each target in order to estimate the NIR emission size; this step is crucial for interpreting the CP results. Sections 4 and 5 contain the most novel aspects of this paper. Here we outline our method for using CPs to probe the amount of skew in the YSO disk emission. Then we apply our method to quantify the "skewed" disk emission and discuss the implications on current disk models. Lastly, in $\S 6$ we treat the special case of HD 45677 and report the first astrometric orbit for the YSO binary MWC 361-A.

\section{OBSERVATIONS AND DATA REDUCTION}

\subsection{Description of IOTA Observations}

All the data presented herein were obtained using the IOTA interferometer (Traub et al. 2003), a long baseline interferometer that observes at visible and NIR wavelengths. Located on Mount Hopkins (Arizona), IOTA is operated by a consortium of institutions, most notably the Smithsonian Astrophysical Observatory and the University of Massachusetts at Amherst. The three $0.45 \mathrm{~m}$ telescopes are movable among 17 stations along two orthogonal linear arms (telescopes $\mathrm{A}$ and $\mathrm{C}$ can move along the $35 \mathrm{~m}$ northeastern arm, while telescope $B$ moves along the $15 \mathrm{~m}$ southeastern arm). By observing a target in many different array configurations, IOTA can synthesize an aperture $35 \times 15 \mathrm{~m}$ (corresponding to an angular resolution of $\sim 5 \times 12$ mas at $1.65 \mu \mathrm{m}$ ).

First results using closure phases have been published by Monnier et al. (2004) and Kraus et al. (2005) using the IONIC3 combiner on IOTA. We only just introduce the IONIC3 combiner here and refer the interested reader to a recent description in Berger et al. (2003); an engineering paper with detailed description of the optical component and its performance is in preparation (J.-P. Berger et al. 2006, in preparation). Light from each telescope is focused into a dedicated single-mode fiber, and the three fibers are aligned using a silicon v-groove array mated to planar waveguides on the integrated optics (IO) device. The optical circuit acts to split the light from each fiber/telescope before recombining each telescope pair $(\mathrm{AB}, \mathrm{BC}, \mathrm{AC})$ at three $\mathrm{IO}$ couplers. This "pairwise" combination scheme leads to six interferometer channels (two for each baseline), and the fringes are detected using a sensitive $\mathrm{HgCdTe}$ (Rockwell PICNIC) detector (Pedretti et al. 2004). Varying coupling efficiencies into the fibers (due to seeing variations and tip-tilt control errors) change the system visibilities to vary in time; however, this effect can be directly calibrated using measurements of the IONIC3 (optical) flux transfer matrix, which relates the six output channels to the three input channels for incoherent light (explained further in Monnier et al. 2004).

The interference fringes are temporally modulated on the detector by scanning piezo mirrors placed in two of the three beams of the interferometer. A typical single observation consists of 200 scans obtained in $\sim 4$ minutes, followed by calibration measurements of the background and single-telescope fluxes (important for characterizing the IO beamsplitters/couplers). Target observations are interleaved with an identical calibration sequence obtained on an unresolved or partially resolved star, serving to calibrate the interferometer's instrumental response and effects of atmospheric seeing on the visibility amplitudes. The target and calibrator sources are typically separated on the sky by $5^{\circ}-10^{\circ}$ and are observed $10-20$ minutes apart; these conditions ensure that the calibrator observations provide a good estimate of the instrument's transfer function.

The target sample was selected from the catalog of Herbig Ae/Be members by Thé et al. (1994). Eleven of the 14 targets come from their Table 1 of bona fide Herbig Ae/Be members or 
TABLE 2

OBSERVING LOG

\begin{tabular}{|c|c|c|c|}
\hline Target & $\begin{array}{l}\text { Date } \\
\text { (UT) }\end{array}$ & Interferometer Configuration $^{\mathrm{a}}$ & Calibrator Names \\
\hline . & 2004 Dec 14 & $\mathrm{~A} 28-\mathrm{B} 10-\mathrm{C} 00$ & HD 27638, HD 27159, HD 26553 \\
\hline MWC 480 & 2002 Dec 13 & A35-B15-C15 & HR 1626 \\
\hline \multirow[t]{7}{*}{ 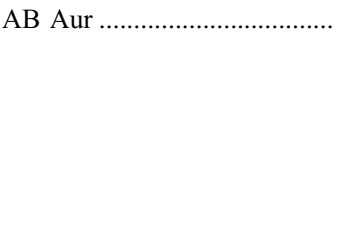 } & 2002 Dec 8-14 & A35-B15-C15 & HR 1626, SAO 57504 \\
\hline & 2002 Dec 16,19 & A35-B05-C10 & HR 1626 \\
\hline & 2003 Feb 19 & & \\
\hline & 2003 Feb 20, 22, 23 & A25-B15-C10 & HR 1626 \\
\hline & 2003 Nov 20,22 & A35-B15-C10 & HR 1626 \\
\hline & 2004 Dec 03 & $\mathrm{~A} 35-\mathrm{B} 15-\mathrm{C} 00$ & HR 1626 \\
\hline & 2004 Dec 13 & A28-B10-C00 & HR 1626 \\
\hline \multirow[t]{4}{*}{ HD 45677 ........................................ } & 2003 Nov 29,30 & A35-B15-C10 & HD 46218 \\
\hline & 2004 Mar 26 & & HD 46218 \\
\hline & 2004 Dec 13, 14 & A28-B10-C00 & HD 46218 \\
\hline & 2004 Dec 16 & A28-B05-C10 & HD 46218 \\
\hline \multirow[t]{2}{*}{ 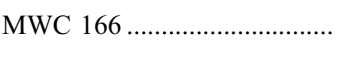 } & 2003 Nov 29-30 & A35-B15-C10 & HD 50067, HD 46128 \\
\hline & 2004 Dec 10 & A35-B15-C00 & HD 47054, HD 49147 \\
\hline 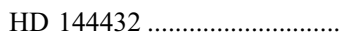 & 2004 Jun 6-8 & A35-B15-C10 & HD 143766 \\
\hline \multirow[t]{2}{*}{ 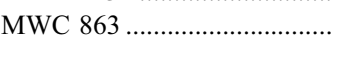 } & 2003 Jun 17,18 & A35-B15-C10 & HR 6153 \\
\hline & 2004 Mar 16, 23-25 & A35-B15-C10 & HR 6153 \\
\hline \multirow[t]{3}{*}{ MWC $275 \ldots \ldots \ldots$} & 2003 Jun 12,21 & $\mathrm{~A} 35-\mathrm{B} 15-\mathrm{C} 10$ & HR 6704, HD 171236 \\
\hline & 2004 Mar 26 & & HR 6704, HD 171236 \\
\hline & 2004 Jun $2,7-8$ & & HD 157546, HD 170657, HD 174596 \\
\hline \multirow[t]{3}{*}{ 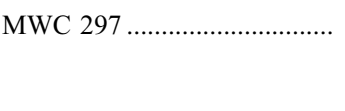 } & 2003 Jun 12,23 & A35-B15-C10 & HD 171236, HR 7149, HR 7066 \\
\hline & 2005 Jun $20,22,26$ & A35-B15-C10 & HR 7066, HR 7149 \\
\hline & 2005 Jun 29,30 & A35-B15-C00 & HR 7066, HD 169268 \\
\hline 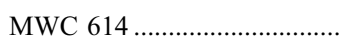 & 2003 Jun $16-17$ & $\mathrm{~A} 35-\mathrm{B} 15-\mathrm{C} 10$ & HD 182101, HD 184502 \\
\hline 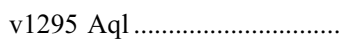 & 2003 Jun 14,17 & A35-B15-C15 & HD 185209 \\
\hline 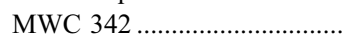 & 2003 Jun 13 & A $35-B 15-C 10$ & HD 191589, HD 199547 \\
\hline \multirow[t]{6}{*}{ 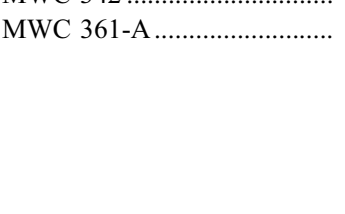 } & 2003 Jun 21,22 & $\mathrm{~A} 35-\mathrm{B} 15-\mathrm{C} 10$ & HR 7967 \\
\hline & 2003 Nov $27-29$ & $\mathrm{~A} 35-\mathrm{B} 15-\mathrm{C} 10$ & HR 7967, HD 197950 \\
\hline & 2004 Jun 6 & A35-B15-C10 & HD 193664 \\
\hline & 2004 Dec 10 & A35-B15-C00 & HR 7967 \\
\hline & 2005 Jun 17,18 & $\mathrm{~A} 35-\mathrm{B} 15-\mathrm{C} 10$ & HD 193664 \\
\hline & 2005 Jun 29 & $\mathrm{~A} 35-\mathrm{B} 15-\mathrm{C} 00$ & HD 193664 \\
\hline \multirow[t]{3}{*}{ 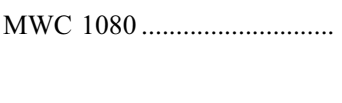 } & 2003 Nov 30 & A35-B15-C10 & HD 221639 \\
\hline & 2004 Dec 10 & $\mathrm{~A} 35-\mathrm{B} 15-\mathrm{C} 00$ & HR 8881 \\
\hline & 2004 Dec 13 & $\mathrm{~A} 28-\mathrm{B} 10-\mathrm{C} 00$ & HR 8881, HD 221639 \\
\hline
\end{tabular}

${ }^{\text {a }}$ Configuration refers to the location of telescopes A, B, and C on the NE, SE, and NE arms, respectively; see $\S 2.1$ for more details.

candidate members. HD 45677 is contained in their Table 3, and MWC 342 is found in their Table $4 \mathrm{a}$; both of these objects are discussed individually in $\S 3.2$. Lastly, we have included one object not listed in the The catalog, RY Tau. RY Tau (F8) is often considered too late-type to be a Herbig Ae/ $\mathrm{Be}$ star and too early to be a T Tauri star. However, the inner disks of T Tauri and Herbig Ae stars seem to share common physics (Millan-Gabet et al. 2006a; Monnier \& Millan-Gabet 2002; Vink et al. 2005), so we have included this target in our sample. We note that the IOTA Interferometer with the IONIC combiner has a limiting $H$-band magnitude of $\sim 7$, and this limitation played a major role in our selections.

The science targets, along with their fundamental parameters, are listed in Table 1. All the science targets presented here were observed using a standard $H$-band filter $\left(\lambda_{0}=1.65 \mu \mathrm{m}, \Delta \lambda=\right.$ $0.30 \mu \mathrm{m}$ ). Full observing information for our science targets can be found in Tables 2 and 3, including dates of observation, interferometer configurations, and calibrator details; note that the calibrators are all nearly unresolved on our baselines and the size uncertainties do not generally significantly contribute to the calibration uncertainties. Figure 1 shows the $u-v$ coverage obtained during each epoch for each science target. In the next section, we discuss our analysis of these data and the techniques used to process raw data into calibrated squared-visibilities $\left(\mathcal{V}^{2}\right)$ and CPs.

\subsection{Data Reduction}

Reduction of the IONIC3 visibility data was carried out using custom software similar in its main principles to that described by Coudé du Foresto et al. (1997) for the FLUOR experiment, developed using IDL. In short, we measure the power spectrum of each interferogram (proportional to the target $\mathcal{V}^{2}$ ) after correcting for intensity fluctuations and subtracting out bias terms from read noise, residual intensity fluctuations, and photon noise (e.g., Perrin 2003). We require that interferograms are detected for at least two of the three baselines in order to ensure a good $\mathrm{CP}$ measurement; this condition is almost always met thanks to a real-time fringe packet "tracker" (discussed further below). Lastly, the data pipeline applies a correction for the variable flux ratios for each baseline by using a flux transfer matrix (e.g., Coudé du Foresto et al. 1997; Monnier 2001). We have studied our calibration accuracy by extensive observations of the binary star $\lambda$ Vir (M. Zhao et al. 2006, in preparation). For bright stars ( $H \lesssim 5 \mathrm{mag}$ ), we have validated $2 \%$ calibration error for $\mathcal{V}^{2}$ (corresponding to $1 \%$ error in visibility). However, most YSOs are faint, requiring slow scan rates and exposing our calibration to seeing changes. Under most conditions for faint stars, we find a calibration error of $\sim 5 \%$ in visibility-squared (e.g., $2.5 \%$ in visibility), 
TABLE 3

Calibrator Information

\begin{tabular}{|c|c|c|c|}
\hline Calibrator Name & Spectral Type & $\begin{array}{l}\text { Adopted Uniform } \\
\text { Disk Diameter } \\
\quad \text { (mas) }\end{array}$ & Reference(s) \\
\hline HD $26553 \ldots \ldots \ldots \ldots$ & A4 III & $0.37 \pm 0.30$ & getCal $^{a}$ \\
\hline HD $27159 \ldots \ldots \ldots \ldots$ & K1 III & $0.30 \pm 0.10$ & getCal \\
\hline HD $27638 \ldots \ldots \ldots \ldots . . . . . . . . .$. & B9 V & $0.32 \pm 0.10$ & getCal \\
\hline 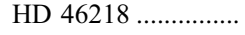 & A5 & $0.36 \pm 0.60$ & getCal \\
\hline HD $47054 \ldots \ldots \ldots \ldots$ & B8 & $0.10 \pm 0.15$ & getCal \\
\hline HD 49147 ................... & B9.5 & $0.35 \pm .03$ & getCal \\
\hline HD $50067 \ldots \ldots \ldots \ldots . . . . . . . .$. & K4 III & $1.35 \pm 0.35$ & getCal \\
\hline HD $143766 \ldots \ldots \ldots . . .$. & F7 V & $0.30 \pm 0.05$ & getCal \\
\hline HD $157546 \ldots \ldots \ldots \ldots . . .$. & B8 V & $0.22 \pm 0.06$ & getCal \\
\hline HD $168415 \ldots \ldots \ldots \ldots . .$. & K4 III & $2.6 \pm 0.3$ & Blackbody fit \\
\hline HD $169268 \ldots \ldots \ldots \ldots . . .$. & F6 III & $0.28 \pm 0.03$ & getCal \\
\hline HD $170657 \ldots \ldots \ldots \ldots . . .$. & $\mathrm{K} 1 \mathrm{~V}$ & $0.58 \pm 0.10$ & getCal \\
\hline HD $171236 \ldots \ldots \ldots \ldots$ & K1 III & $1.0 \pm 0.5$ & getCal \\
\hline HD $174596 \ldots \ldots \ldots \ldots$ & A3 V & $0.2 \pm 0.05$ & getCal \\
\hline HD $182101 \ldots \ldots \ldots \ldots$ & F6 V & $0.4 \pm 0.1$ & getCal \\
\hline HD $184502 \ldots \ldots \ldots \ldots . . .$. & B3 III & $0.19 \pm 0.10$ & getCal \\
\hline  & K3 III & $0.7 \pm 0.2$ & Blackbody fit \\
\hline HD 191589 .................. & K5 III & $1.47 \pm 0.37$ & getCal \\
\hline HD $193664 \ldots \ldots \ldots \ldots . . . .$. & G0 V & $0.22 \pm 0.07$ & getCal \\
\hline HD $197950 \ldots \ldots \ldots \ldots . .$. & $\mathrm{A} 8 \mathrm{~V}$ & $0.32 \pm 0.06$ & getCal \\
\hline HD $199547 \ldots \ldots \ldots \ldots . .$. & K0 III & $0.75 \pm 0.15$ & getCal \\
\hline HD $221639 \ldots \ldots \ldots . . .$. & $\mathrm{K} 1 \mathrm{~V}$ & $0.51 \pm 0.10$ & getCal \\
\hline HR $1626 \ldots \ldots \ldots \ldots \ldots . . . . . . . . .$. & K0 III & $1.2 \pm 0.7$ & getCal \\
\hline 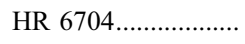 & K0 III & $1.89 \pm 1.47$ & getCal \\
\hline HR 7066................... & K0I & $2.17 \pm 1.01$ & getCal \\
\hline 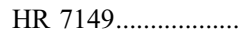 & K2 III & $1.69 \pm 0.75$ & getCal \\
\hline HR 7967..................... & G8 III & $0.76 \pm 0.30$ & getCal \\
\hline  & M2 III & $2.4 \pm 0.5$ & van Belle (1999) \\
\hline SAO $57504 \ldots \ldots \ldots \ldots$ & K0 & $0.58 \pm 0.36$ & getCal \\
\hline
\end{tabular}

${ }^{a}$ getCal is maintained and distributed by the Michelson Science Center (http://msc.caltech.edu).

although errors of $\gtrsim 10 \%$ are occasionally encountered during poor seeing conditions. For very resolved objects, the calibration error is dominated by imperfect subtraction of the power spectrum background, resulting in a $\Delta \mathcal{V}^{2}=0.02$ noise floor. To be conservative, we adopted a uniform $5 \%$ calibration error (with $\Delta \mathcal{V}^{2}=$ 0.02 noise floor) for all $\mathcal{V}^{2}$ in this paper, although this overestimates the error for many epochs (Millan-Gabet et al. [2006c] adopted similar values for $\mathrm{AB}$ Aur).

In order to measure the closure phase, a fringe-tracking algorithm was applied in real time while recording interferograms (Pedretti et al. 2005), ensuring that interference occurs nearly simultaneously for all baselines. We followed the method of Baldwin et al. (1996) for calculating the complex triple amplitude in deriving the closure phase, explicitly guaranteeing that the fringe frequencies for each triple product also "close" $\left(\nu_{\mathrm{AB}}+\nu_{\mathrm{BC}}+\nu_{\mathrm{CA}}=0\right)$. One calibration step of Baldwin et al. (1996) we do not need to apply is a photon-noise bias correction to the triple product (e.g., Wirnitzer 1985), which is only necessary for "all-in-one" combiners.

Pairwise combiners (such as IONIC3) can have a large instrumental offset for the closure phase, which is generally calibrated by using a point-source calibrator. The instrumental closure phase of IONIC 3 drifts less than $1^{\circ}$ over many hours (a remarkable fact given the unstabilized IOTA environment whose temperature can drift by $>10 \mathrm{~K}$ during a night), owing to the miniature dimensions of the IO component. Also, chromaticity effects limit our absolute precision when the calibrator and target are not of the same spec- tral type due to different effective wavelengths when using the broadband $H$ filter. Recent engineering tests indicate that the instrumental closure phase $\left(\Phi_{\mathrm{CP}}\right)$ of the current IONIC3 combiner varies little $(\leqslant 0.5)$ between a hot star (B8) and a cool star (M3) when using the broadband $H$ filter. We minimize these errors through repeated calibration of our targets with calibrators of similar spectral type. In light of this, we have adopted a systematic error of $\Delta \Phi_{\mathrm{CP}}=0.5$ for the measurements presented here to represent the residual correction for wavelength dependence. This error is not applied in our analysis (it is not random and should be fixed for each target/calibrator pair) but rather establishes a minimum CP magnitude in order to be confident of detecting "intrinsic" nonzero closure phase (i.e, skew) in a target. As a cross-check, we note that our targets are generally redder than our calibrators, yet we have found no systematic offset in our measured YSO closure phases, consistent with expectations from the engineering study. Lastly, we established the sign of our closure phase (baseline triangle connecting telescopes $\mathrm{A} \rightarrow \mathrm{B} \rightarrow \mathrm{C} \rightarrow \mathrm{A}$ ) using the test measurements of Capella and Matar in comparison to published orbits (Hummel et al. 1994, 1998).

In Figure 2 we present all the $\mathcal{V}^{2}$ data from this survey work, where each epoch of observation is denoted by a unique plot symbol. For this and subsequent data analysis, the $\mathcal{V}^{2}$ data from a given configuration has been averaged in the $u-v$ plane (on the scale of $u-v$ distance $4 \mathrm{~m}$ ) to improve signal-to-noise ratio. We have compared our $\mathcal{V}^{2}$ results with those from previous workers and found good agreement within quoted uncertainties. Note that we report here the first ever infrared interferometry results for the targets MWC 342 and HD 45677.

We present all the CP results in Figures 3 and 4, where the data have been plotted against the hour angle of the observation and the array configuration is noted in the legend. As was done for the $\mathcal{V}^{2}$ data, the CP data was averaged in the $u-v$ (hyper)plane before plotting.

All $\mathcal{V}^{2}$ and $\mathrm{CP}$ data are available in both averaged and unaveraged form from the authors; ${ }^{9}$ all data products are stored in the FITS-based, optical interferometry data exchange format (OI-FITS), recently described in Pauls et al. (2005).

\section{ANALYSIS OF VISIBILITY DATA}

\subsection{SED Decomposition and Size Estimation}

In this section, we carry out the (now) standard procedure of YSO size estimation, first outlined by Millan-Gabet et al. (2001). While most of the targets have already been observed by either IOTA, PTI, or Keck, we have much better $u-v$ coverage than previous studies, as well as a few new targets ( MWC 342, HD 45677), necessitating a fresh visibility and disk size analysis.

Visibilities from YSOs are traditionally interpreted by fitting simple emission models, such as Gaussians and rings (e.g., Millan-Gabet et al. 2001; Monnier \& Millan-Gabet 2002), after correcting for the unresolved stellar emission. For each target, we have collected recent visible and near-infrared photometry from the literature to create a SED (see Table 1 for a list of specific photometry references for each object). Using the appropriate Kurucz model (Kurucz 1979) for each star (from literature spectral type), we fit the SED as a combination of the star plus dust, the latter being simply modeled as a single-temperature blackbody; the reddening is another free parameter of this model important to match the blue/visible portion of the spectrum. After fitting, we

\footnotetext{
${ }^{9}$ Currently, all data can be found at the data archives section of the Optical Long Baseline Interferometry News (OLBIN) Web site, http://www.olbin.jpl. nasa.gov.
} 


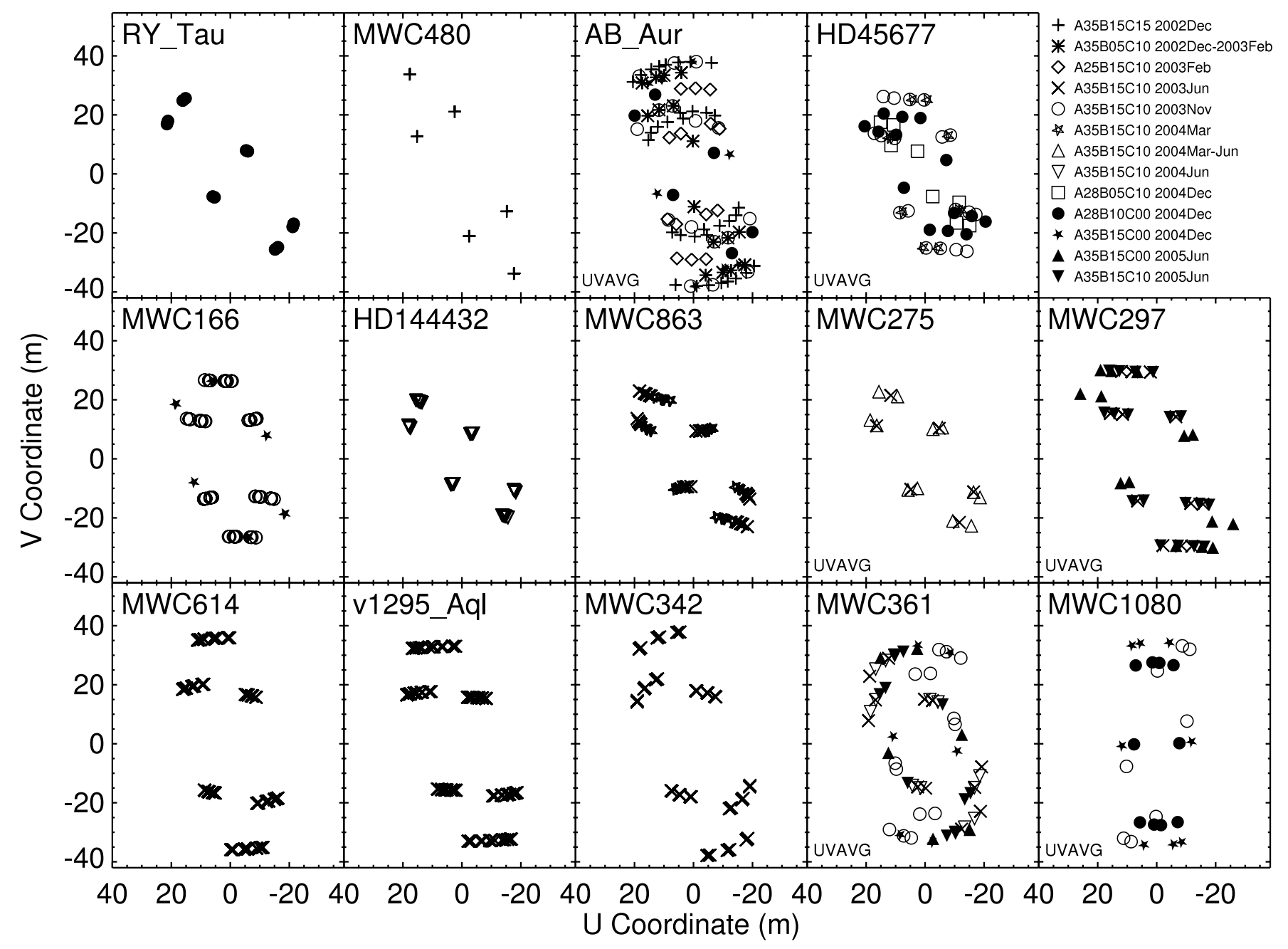

FIG. 1.- These panels show the $u-v$ coverage of the data for all targets presented in this paper. Each observing configuration and epoch are denoted by a unique


presentation clarity, and these panels are denoted by the word "UVAVG" in the bottom left corner.

can estimate the fraction of flux at $1.65 \mu \mathrm{m}$ arising from the dust component compared to the star. Because YSOs are photometrically variable, we included photometry from multiple epochs to illustrate the level of variability, and this uncertainty dominates the decomposition error budget. Figures 5-18 show the results of the SED decompositions, and our estimates of the dust fraction (with errors) are tabulated in Table 4. A more detailed description of this algorithm can be found in Monnier et al. (2005).

Next, the $\mathcal{V}^{2}$ data are fit using a two-component model consisting of the unresolved stellar source (flux fraction constrained from SED fitting) and a thin circularly symmetric ring of NIR emission (Gaussian cross section, $25 \%$ thickness). ${ }^{10}$ In some cases, a fully resolved extended component ("halo") was included if evident from the data. These results are found in Table 4 and the right panels of Figures 5-18. Note that this halo need not be of the kind described by Vinkovic et al. (2006) but could arise from scattering off the surface of the disk, or from localized emission within even a few AU of the star (Millan-Gabet et al. 2006c).

${ }^{10}$ While our new observations have better $u$-v coverage than previous work, the relatively short baselines of IOTA coupled with the $5 \%$ calibration errors on the visibility-squared forbid us from definitively detecting large-scale elongations (for most sources), such has been reported using the longer baseline Palomar Testbed Interferometer (e.g., Eisner et al. 2004).
In order to interpret the presence or absence of halos, one must understand the field of view of modern infrared interferometers. The most important limitation on field of view (FOV) is restricted by the field of an individual telescope. For fiber-optic based systems (Keck, PTI, IOTA3-IONIC), this FOV corresponds to the diffraction-limited beam, which is 1 ". 0 for IOTA3-IONIC at $H$ band, 1.4 for PTI at $K$ band, and 0.055 for Keck at $K$ band. Note the original IOTA2 experiment (Millan-Gabet et al. 2001) had a "freespace" combiner, which had a $2^{\prime \prime}-3^{\prime \prime}$ FOV. Thus, light beyond this scale does not make it into the beam combiner and is completely neglected. The "interferometric" FOV is usually limited by bandwidth-smearing. This means that light farther than $\lambda^{2} / \Delta \lambda B$ ( $B$ is the interferometer baseline, $\lambda$ is the central observing wavelength, and $\Delta \lambda$ is the bandwidth) from the central source becomes incoherent and does not produce fringes at the phase center (zero optical path length difference). In practice, this is about 6 times the fringe spacing, corresponding to about 60 mas for IOTA ( $H$ band), 25-30 mas for PTI and Keck ( $K$ band). In this latter case, light outside the interferometric FOV is detected but is not coherent and acts to lower the observed visibility. See Monnier (2003) for further discussion of modern optical and infrared interferometry.

Before discussing individual objects, we note that evidence for large-scale (0.01-0.50) halos was found for AB Aur (as found by Millan-Gabet et al. 2006c), MWC 275, MWC 297, and 


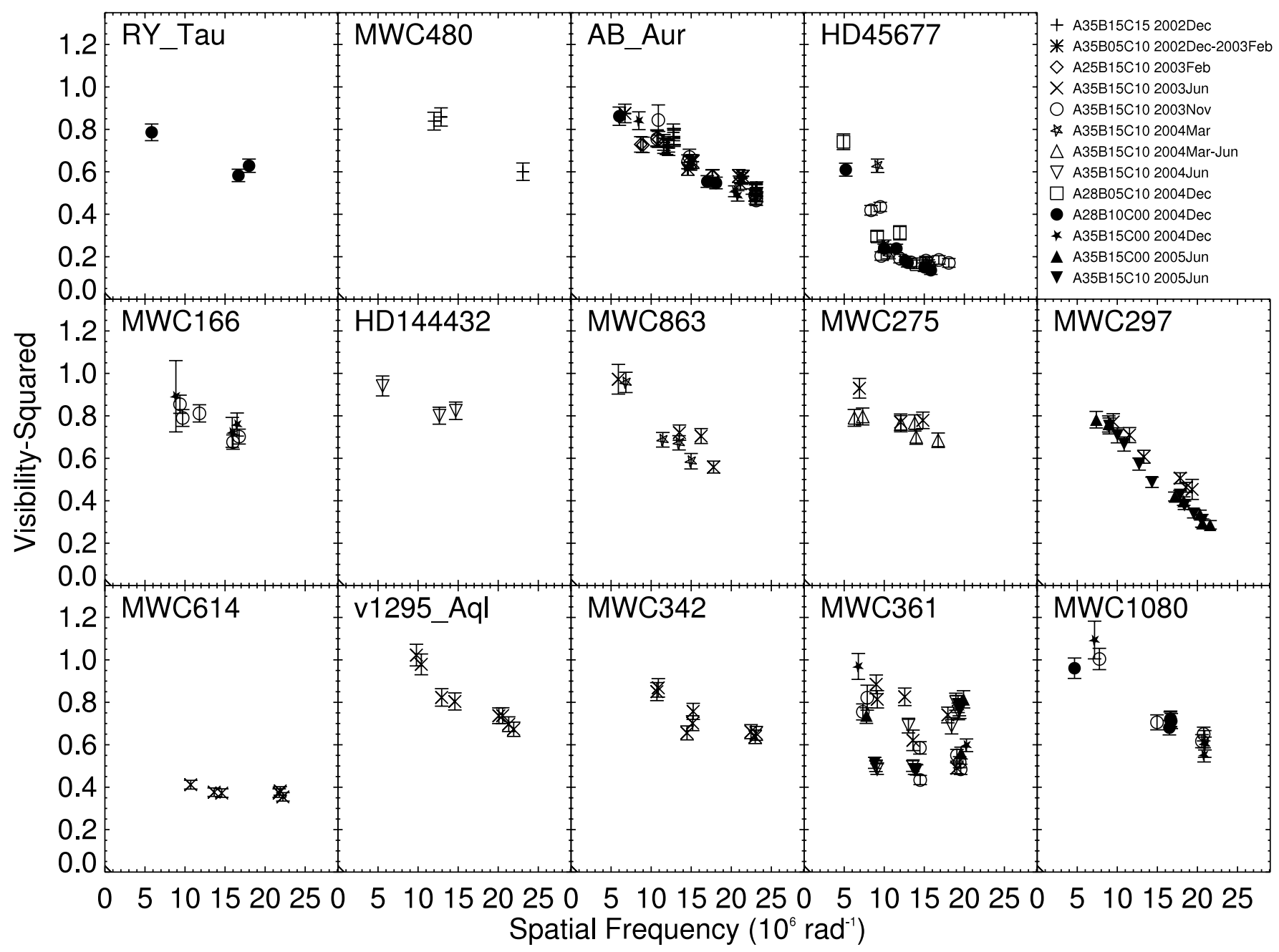

FIg. 2.-These panels show the $\mathcal{V}^{2}$ results as a function of spatial frequency for all targets presented in this paper. Each observing configuration and epoch are denoted by a unique plotting symbol according to the legend in the top right panel.

RY Tau. Also, we surprisingly found that the NIR disk emissions for HD 45677 and MWC 614 were completely resolved by the $38 \mathrm{~m}$ IOTA baselines; this allowed a unique comparison of the "predicted" stellar flux contribution (from SED fitting) with the observed values, as well as search for the tell-tale signature of "ringlike" emission compared to more smooth "Gaussian-like" emission. We discuss unique aspects of some targets below.

\subsection{Individual Objects}

Many objects need special attention and specific discussion. In this section we briefly describe unusual and interesting characteristics of targets in our sample.

RY Tau.-This star is variously spectral-typed as K1 (Akeson et al. 2005b) to F8 (Mora et al. 2001), an intermediate object between the Herbig Ae/Be and classical T Tauri stars. Adopting F8 and fitting to the SED, we find a stellar luminosity of $\sim 5 L_{\odot}$, a few times lower than that reported by other workers. The quality of the RY Tau visibility data is particularly excellent owing to good seeing, and we take seriously the low-visibility data point at short baselines seen in Figure 5. In this figure, we present fits assuming either a $10 \%$ extended halo emission or no halo emission (both results are also reported in Table 4). Clearly, halo emission fits the data much better and gives a ring diameter consistent with $K$-band results of Akeson et al. (2005b), ring diameter $2.60 \pm$ 0.14 mas, although this high level of $H$-band extended emission seems incompatible with their reported $2 \%$ scattered light at $0.9 \mu \mathrm{m}$.

$A B$ Aur.-This object was discussed in detail in Millan-Gabet et al. (2006c). Here we present an independent size analysis but use the same IOTA data set.

$H D$ 45677.- - Here we report the first long-baseline interferometry data for this target. To aid interpretation, we obtained recent visible photometry from the MDM Observatory in 2004 November and used NIR photometry from 2MASS. Surprisingly, the disk emission appears fully resolved at the longest IOTA baselines, a direct measurement of $62 \% \pm 3 \%$ dust fraction, in reasonable agreement with the value derived from the SED decomposition $(65 \% \pm 15 \%)$.

Interestingly, the visibility data do not show the expected shape for ringlike emission (specifically, large "ringing" at long baselines due to the ring thinness). Although we were able to find an acceptable fit for a ring model, the apparent constant visibilities at long baselines motivated additional fitting of a Gaussian emission model, and this result, along with the best-fit ring model, is included in Table 4 and Figure 8. As is obvious from this figure, additional data at slightly longer baselines can rule out or definitively establish ringlike emission (as opposed to smooth, Gaussian emission) for this object. The distinction may be critical to decide between envelope and inner rim models of the NIR emission (e.g., Vinković et al. 2006). 


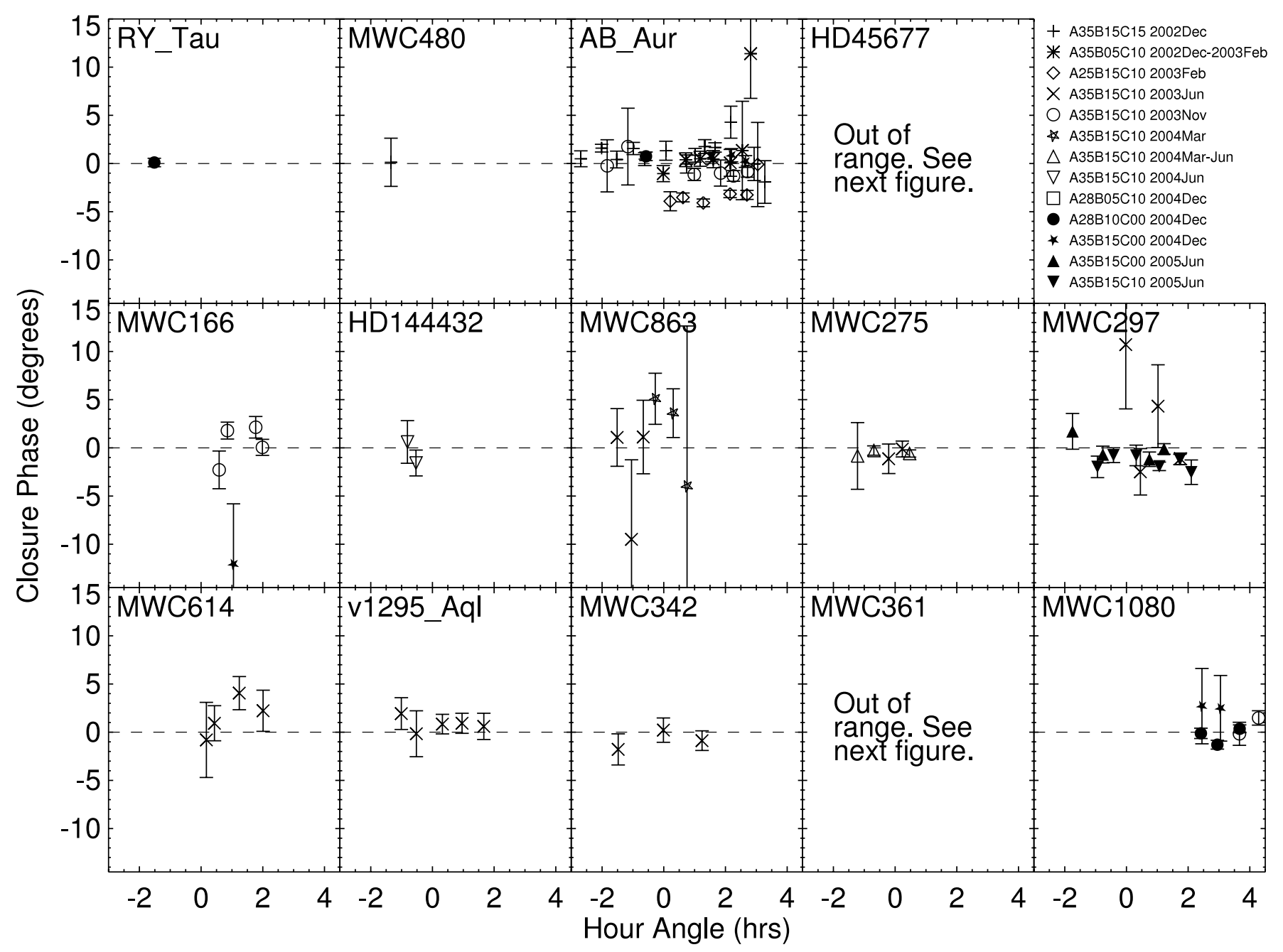

FIG. 3.-Observed IOTA3 closure-phase results as a function of hour angle for all targets presented in this paper. Each observing configuration and epoch are denoted by a unique plotting symbol according to the legend in the top right panel. Note that the closure phases for HD 45677 and MWC 361-A are too large to display here and are included in Fig. 4.
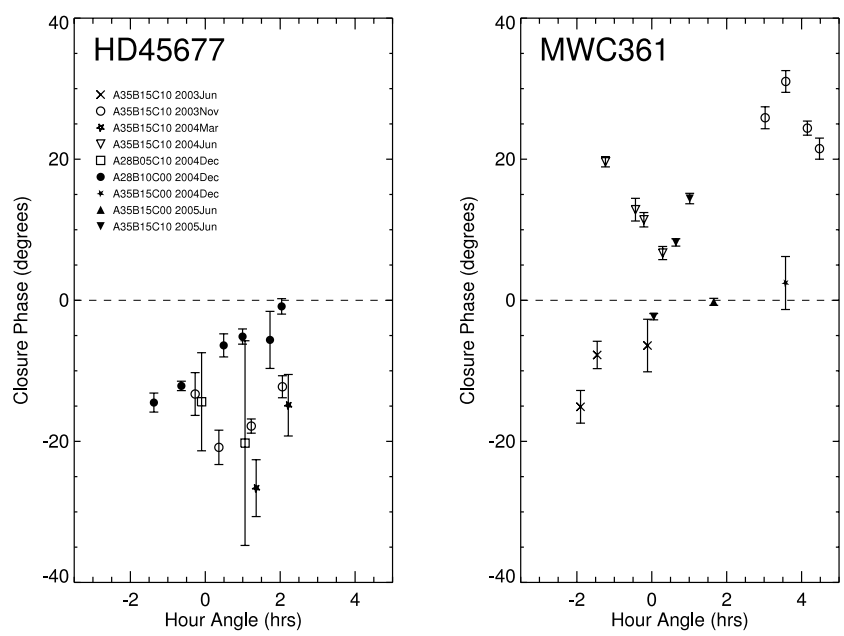

FIG. 4.-Observed IOTA3 closure-phase results as a function of hour angle for HD 45677 and MWC 361-A. Each observing configuration and epoch are denoted by a unique plotting symbol according to the legend in the left-hand panel.
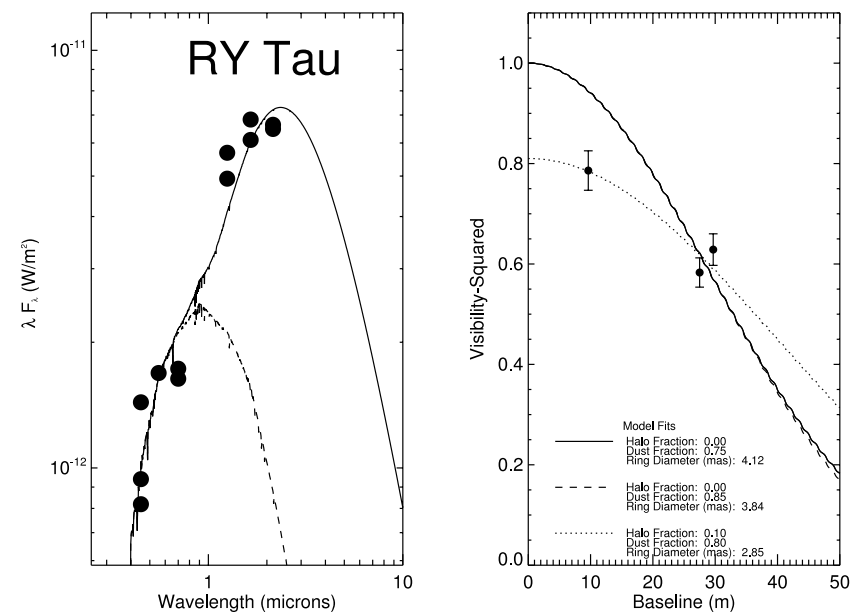

FIG. 5.-Left: SED for RY Tau, including a simple two-component fit. The dashed line represents the stellar contribution only, and the solid line includes blackbody dust emission. Photometry references are included in Table 1. No error bars are shown because intrinsic variability dominates over measurements uncertainty at any given epoch. Right: Visibility data are presented along with best-fit ring models exploring different values for halo and dust fractions (see inset legend for details). 

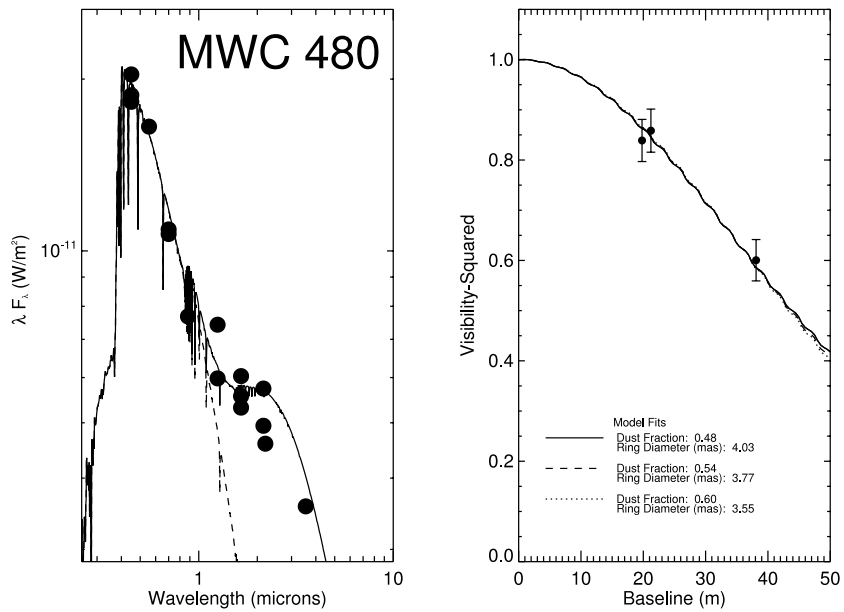

FIG. 6.-Left: SED for MWC 480, including a simple two-component fit. The dashed line represents the stellar contribution only, and the solid line includes blackbody dust emission. Photometry references are included in Table 1. No error bars are shown because intrinsic variability dominates over measurements uncertainty at any given epoch. Right: Multiple estimates for the dust fraction are used to constrain ring model fits to the IOTA3 visibility data.

Here we are first to note that the emission appears quite elongated, and we also find large nonzero closure phases. This target is clearly very different from the others in our sample, and a more complex model is developed in $\S 6.1$ after a more general discussion of closure phases.

MWC 166.-Millan-Gabet et al. (2001) found that there is very little, if any, IR excess at $H$ band $(12 \% \pm 20 \%)$ for this highluminosity target (B0). However, these workers did find the longbaseline visibility to be resolved ( $V \sim 90 \%$ ), after correcting for $22 \%$ emission from a nearby companion (also B0 spectral type at separation $\sim 0$ "'6; Corporon 1998). We have obtained new visible photometry at MDM Observatory, which, when combined with 2MASS data, also suggests a small IR excess $(10 \% \pm 10 \%)$.

Our new visibility data also confirm the slightly resolved visibility at long baselines ( $V \sim 85 \%$ ), although this is before correction for the known companion. Because the IONIC3 combiner uses single-mode fibers (unlike the original IOTA two-telescope
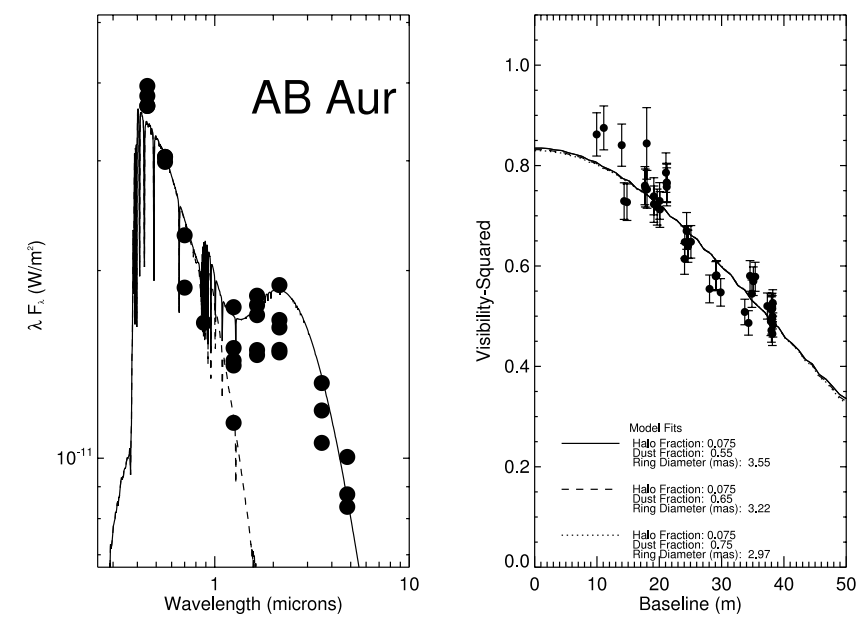

FIG. 7.-Left: SED for AB Aur, including a simple two-component fit. The dashed line represents the stellar contribution, and the solid line includes blackbody dust emission. Photometry references are included in Table 1. No error bars are shown because intrinsic variability dominates over measurements uncertainty at any given epoch. Right: Multiple estimates for the dust fraction are used to constrain ring model fits to the IOTA3 visibility data; here we also include $7.5 \%$ emission from an extended halo to fit short baseline data.


FIG. 8.-Left: SED for HD 45677, including a simple two-component fit. The dashed line represents the stellar contribution, and the solid line includes blackbody dust emission. Photometry references are included in Table 1. Because the SED has changed significantly for this target in recent years, we only include most recent photometry (see Table 1 for references). Right: Here we fitted the IOTA3 visibility with a symmetric ring model, as well as a Gaussian emission model. Large residuals here are a sign of elongated asymmetric emission, and a more complicated model is presented in Fig. 24.

survey), the companion flux is more attenuated than the primary star, although the exact amount is difficult to predict (depending on the seeing and the optical alignment). At short baselines $(15 \mathrm{~m})$, the observed visibility is $\sim 90 \%$ - this limits the companion's contribution to $\leq 10 \%$ of the total $H$-band flux. In order to account for this, we performed fits including both a $0 \%$ and $10 \%$ contribution from the companion, while also constraining the dust fraction to be between $1 \%$ and $20 \%$. The allowed ring diameters span the range 4-15 mas, with a best-fit for $80 \%$ primary star, $4 \%$ secondary star (incoherent flux), and $16 \%$ disk emission using a ring diameter of 7.5 mas. Some representative fits are shown in Figure 9.

$M W C$ 863.-A binary companion was detected by Corporon (1998), a T Tauri star (K4) at 1."1 and $\Delta H \sim 2.24$. We see no
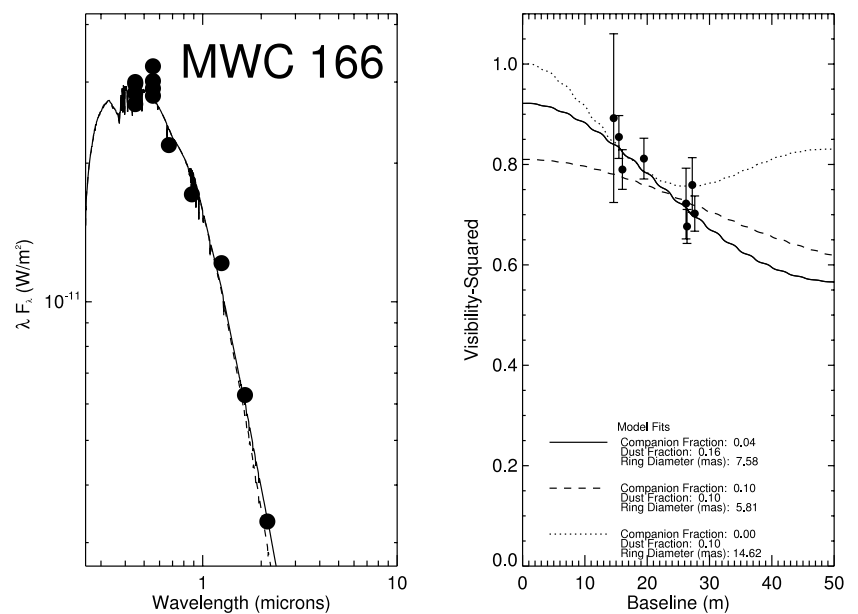

FIG. 9.-Left: SED for MWC 166, including a simple two-component fit. The dashed line represents the stellar contribution, and the solid line includes blackbody dust emission. Photometry references are included in Table 1. No error bars are shown because intrinsic variability dominates over measurements uncertainty at any given epoch. Right: Because of a known, nearby companion that is completely resolved in our observations, the visibility does not go to unity at the origin. Multiple estimates for the companion contribution and dust fraction are used to constrain ring model fits to the IOTA3 visibility data. 

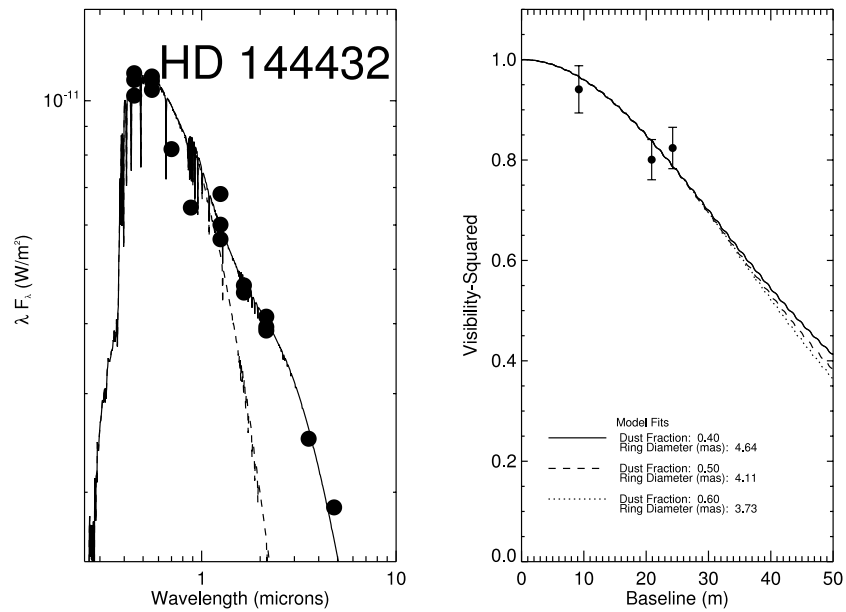

FIG. 10.-Left: SED for HD 144432, including a simple two-component fit. The dashed line represents the stellar contribution, and the solid line includes blackbody dust emission. Photometry references are included in Table 1. No error bars are shown because intrinsic variability dominates over measurements uncertainty at any given epoch. Right: Multiple estimates for the dust fraction are used to constrain ring model fits to the IOTA3 visibility data.

evidence for this companion in our visibility data, since the field of view of an IONIC3 single-mode fiber is only \pm 0 " 5 . Although the companion flux was taken into account in our estimate of the dust fraction in SED fitting, the SED fit is still poor in the NIR region. While our measured $\mathcal{V}^{2}$ is similar to that found by MillanGabet et al. (2001), our ring diameter estimate is much smaller because we did not apply the same visibility "correction" to compensate for the incoherent flux of the companion (our result here is consistent with the recent result using the longer baseline Keck Interferometer at $K$ band; Monnier et al. 2005). Also, our luminosity determination is significantly lower here than that recently reported in Monnier et al. (2005), due to selection of more recent optical photometry. Better coeval photometry is needed for this target in order to estimate stellar and disk properties precisely.

MWC 297.- $\mathcal{V}^{2}$ data are better fit using a 5\% extended halo emission, although the fit is reasonable when assuming no halo
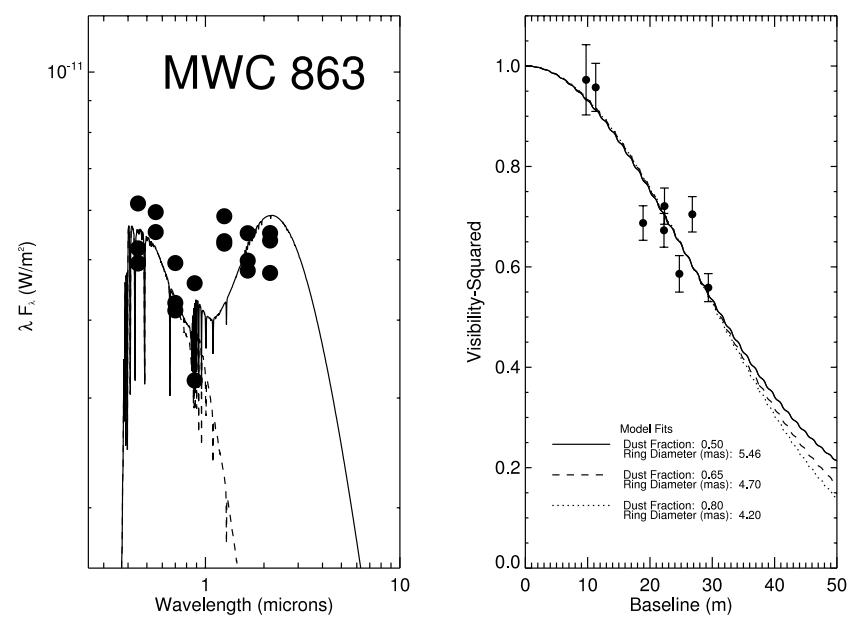

FIG. 11.-Left: SED for MWC 863, including a simple two-component fit, after removing flux from the T Tauri companion (Corporon 1998). The dashed line represents the stellar contribution only, and the solid line includes blackbody dust emission. Photometry references are included in Table 1. No error bars are shown because intrinsic variability dominates over measurements uncertainty at any given epoch. Right: Visibility data are presented along with best-fit ring models covering a large uncertainty range in fraction of $H$-band emission arising from disk
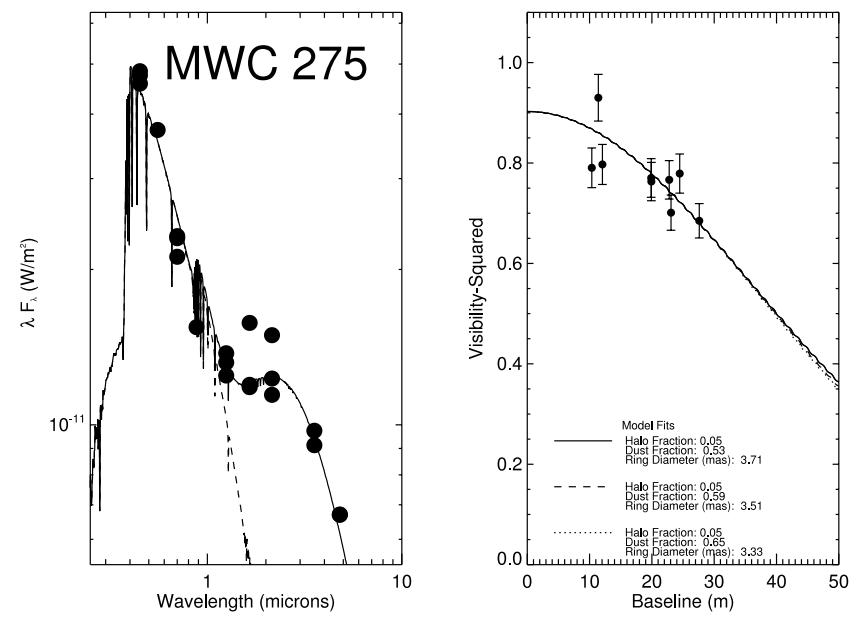

FIG. 12.-Left: SED for MWC 275, including a simple two-component fit. The dashed line represents the stellar contribution, and the solid line includes blackbody dust emission. Photometry references are included in Table 1. No error bars are shown because intrinsic variability dominates over measurements uncertainty at any given epoch. Right: Multiple estimates for the dust fraction are used to constrain ring model fits to the IOTA3 visibility data; here we include $5 \%$ emission from extended halo to fit shortest baselines.

(see Fig. 13). The best-fit values (and uncertainty estimates) in Table 4 include a $2.5 \% \pm 2.5 \%$ estimate for halo emission. This is one of the most resolved disks in our survey, and, while our $u-v$ coverage is limited, there is slight indication of elongation along position angle (P.A.) $\sim 100^{\circ}$; more data are needed to confirm this.

$M W C$ 614.-The nearly flat visibility curve indicates the dust emission is entirely resolved even at short baselines and that the star makes up $61 \%$ of the flux, consistent with an SED decomposition estimate of $63 \% \pm 10 \%$. We can convert this to a lower limit on the dust envelope size, ring diameter $\gtrsim 13$ mas (best-fit Gaussian FWHM 15.9 mas; see Fig. 14). However, as for HD 45677, we see no telltale sign in the $\mathcal{V}^{2}$ data for a ringlike structure that has the distinctive Bessel function shape in Fourier space (e.g., Gaussian profile is a better fit than ring profile). This target would be an ideal source for adaptive optics on an $8 \mathrm{~m}$ class telescope. The large ring size derived here is
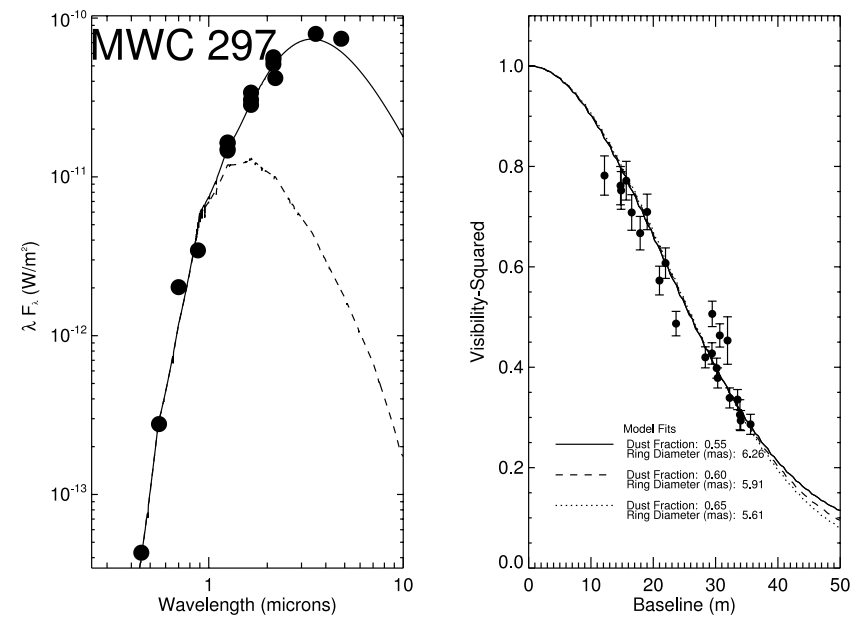

FIG. 13.-Left: SED for MWC 297, including a simple two-component fit. The dashed line represents the stellar contribution only, and the solid line includes both star and blackbody dust emission. Photometry references are included in Table 1. No error bars are shown because intrinsic variability dominates over measurements uncertainty at any given epoch. Right: Multiple estimates for the dust fraction are used to constrain ring model fits to the IOTA3 visibility data. 

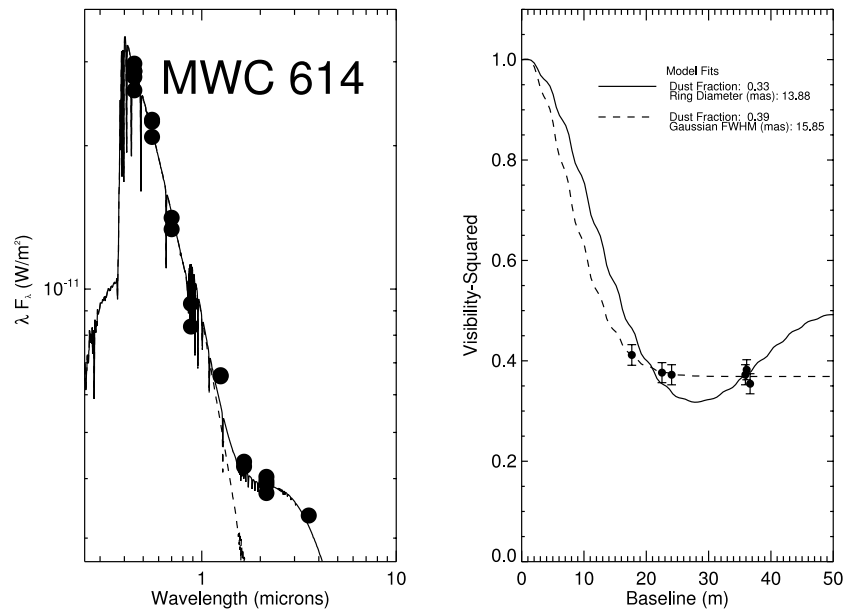

FIG. 14.-Left: SED for MWC 614, including a simple two-component fit. The dashed line represents the stellar contribution only, and the solid line includes blackbody dust emission. Photometry references are included in Table 1. No error bars are shown because intrinsic variability dominates over measurements uncertainty at any given epoch. Right: Visibility data are presented along with the best-fit ring and Gaussian models.

anomalous for the Herbig Ae size-luminosity relation (Monnier et al. 2005). This could be partially explained if there is a stellar companion within about $1^{\prime \prime}$ of the primary star, since this would lead to an overestimate of the dust ring size in our study.

$M W C$ 342.-We present first long-baseline interferometry data on this target. While our data indicate elongated emission, our very limited data set does not allow us to meaningfully quantify this. We note that the evolutionary state of MWC 349 is unclear; Miroshnichenko et al. (1999a) suggest that this star, as well as HD 45677, are possibly X-ray binaries like CI Cam.

MWC 361-A.-A 6" companion to MWC 361-A was first discovered by Li et al. (1994). While this object does not affect our measurements, the close companion (separation $\sim 18$ mas) discovered by Millan-Gabet et al. (2001) obviously is easily detected and appears to be related to the $\sim 3.7 \mathrm{yr}$ periodic variations in the $\mathrm{H} \alpha$ emission seen by Miroshnichenko et al. (1998). Indeed, Pogodin et al. (2004) recently reported a detailed spec-
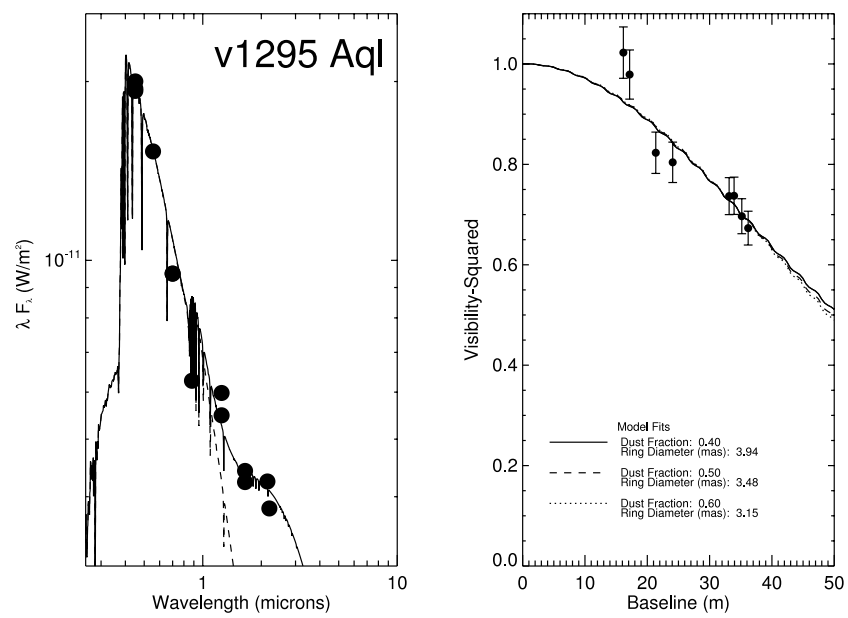

FIG. 15.-Left: SED for v1295 Aql, including a simple two-component fit, after removing flux from the T Tauri companion (Corporon 1998). The dashed line represents the stellar contribution only, and the solid line includes blackbody dust emission. Photometry references are included in Table 1 . No error bars are shown because intrinsic variability dominates over measurements uncertainty at any given epoch. Right: Visibility data are presented along with best-fit ring models covering a large uncertainty range in fraction of $H$-band emission arising from disk.
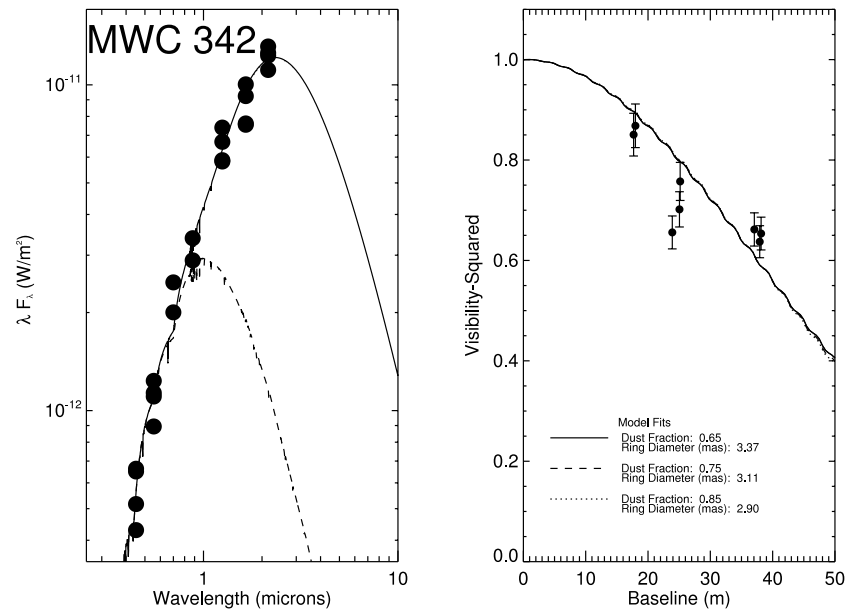

FIG. 16.-Left: SED for MWC 342, including a simple two-component fit. The dashed line represents the stellar contribution only, and the solid line includes blackbody dust emission. Photometry references are included in Table 1 . No error bars are shown because intrinsic variability dominates over measurements uncertainty at any given epoch. Right: Multiple estimates for the dust fraction are used to constrain ring model fits to the IOTA3 visibility data. The poor fit might indicate elongated emission; however, our limited data set does not allow further investigation at this time.

troscopic orbit (more discussion of this in $\S 6.2$ ). Here in Figure 17, we have plotted the $\mathcal{V}^{2}$ of the primary star after correcting for the fainter companion contribution (made possible only after the full orbit analysis of $\S 6.2$ ). Note that this ring diameter estimate assumes the companion star is itself unresolved, a reasonable assumption based on the observed size-luminosity relations. Interestingly, the NIR ring diameter found here is undersized compared to the normal size-luminosity relation for Herbig Ae/ late Be stars, as also found for some other early Be Herbigs (Monnier \& Millan-Gabet 2002; Eisner et al. 2004).

$M W C$ 1080.-There seems to be a fair amount of uncertainty on the spectral type of this star, ranging from $\mathrm{B} 0-2$ (Cohen \&
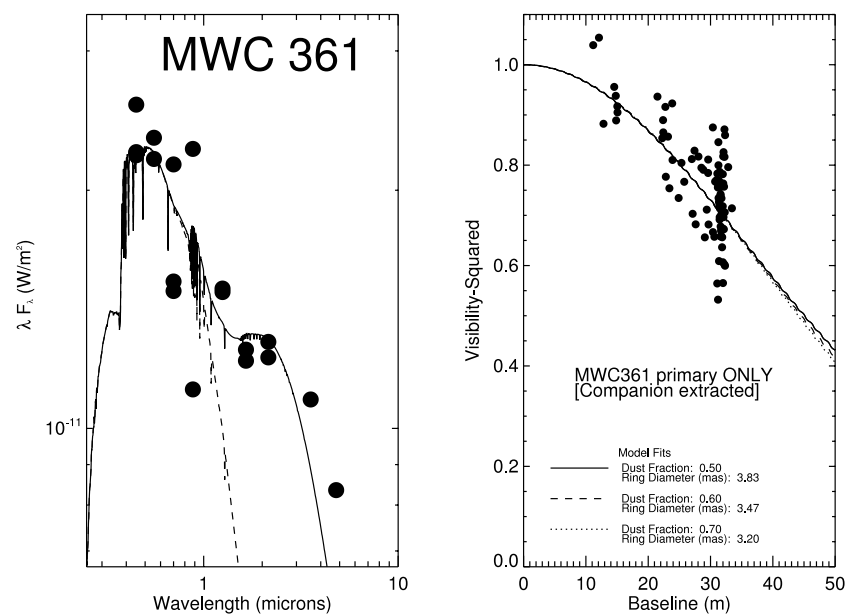

FIG. 17.-Left: SED for MWC 361-A, including a simple two-component fit. The dashed line represents the stellar contribution only, and the solid line includes blackbody dust emission. Photometry references are included in Table 1. No error bars are shown because intrinsic variability dominates over measurements uncertainty at any given epoch. Right: Here we present the corrected visibility data of the primary component after removing the close binary companion. No formal error bars are shown since the extraction process introduces an uncertain error; however, the scatter in values should represent the uncertainty. Visibility fits are presented along with best-fit ring models covering a large uncertainty range in fraction of $H$-band emission arising from the disk (based on SED decomposition). 

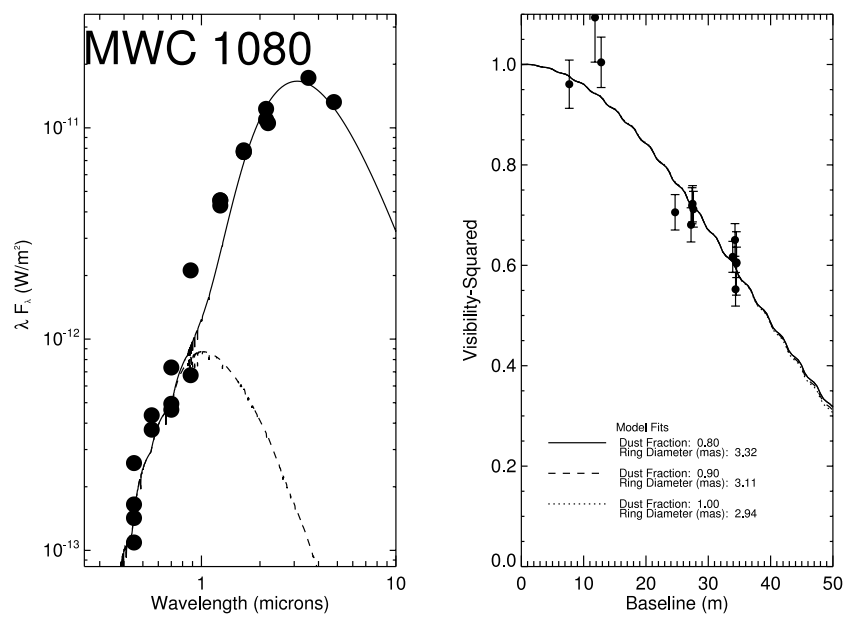

FIG. 18.-Left: SED for MWC 1080, including a simple two-component fit. The dashed line represents the stellar contribution only, and the solid line includes blackbody dust emission. Photometry references are included in Table 1. No error bars are shown because intrinsic variability dominates over measurements uncertainty at any given epoch. Right: Visibility data are presented along with best-fit ring models covering a large uncertainty range in the fraction of $H$-band emission arising from disk.

Kuhi 1979) to A0-3 (Yoshida et al. 1992), and distances of $1-2.5 \mathrm{kpc}$. Here we adopt the earlier spectral type and a distance of $2.2 \mathrm{kpc}$ (Grankin et al. 1992). In principle, our visibility data should be sensitive to the "wide" binary (Corporon 1998) seen with a separation of $\sim 0$ ".8 $8(\Delta H \sim 2.8)$; however, there is no sign of this in our data, presumably due to a high brightness ratio and relatively large off-axis distance.

\section{CLOSURE PHASE AS TOOL FOR STUDYING YSOs}

\subsection{Background}

Basic interferometry theory informs us that an interference fringe measured on an individual baseline corresponds to a single Fourier component of the brightness distribution under scru- tiny. Basic Fourier theory further informs us that any brightness distribution can be reconstructed by collection of sufficient Fourier components (i.e., "visibility" data). Thus, an "imaging" interferometer, such as the radio Very Large Array (VLA), is characterized by a large number of telescopes (corresponding to many simultaneous Fourier components) and a range of baselines (for imaging a range of spatial scales). While the VLA accomplishes this using 27 telescopes with a maximum spacing/minimum spacing of $\gtrsim 100$, current infrared interferometers have three to six telescopes with maximum/minimum spacings of $\lesssim 10$. Not surprisingly, current interferometers have difficulty performing modelindependent image reconstructions (except for simple scenes such as binary stars).

The problem is made more vexing for optical interferometry since atmospheric turbulence scrambles the Fourier "phases" of the observed interference fringes. In some cases, these Fourier (fringe) phases are not important-it can be easily proven that "centrosymmetric" images can be completely characterized by the fringe amplitudes alone. ${ }^{11}$ From this perspective, fringe phases tell us about asymmetric information in an image and, as such, are unnecessary for characterizing simple objects such as (idealized) stellar photospheres or ellipsoidal distributions (such as flat inclined disks).

\subsection{What Is the Closure Phase?}

The closure-phase method was developed by early radio interferometrists (Jennison 1958) to calibrate phase drifts in phasecoherent (heterodyne) detection systems, and it was first applied to optical data in the context of aperture masking (Haniff et al. 1987). The basic idea can be explained by considering a threetelescope array, as pictured in Figure 19. Consider that turbulent air pockets above aperture 2 will cause equal but opposite effects on the two baselines connecting apertures 1-2 and apertures $2-3$. Thus, summing up phases around a triangle of three telescopes

${ }^{11}$ By defining the coordinate origin at the center of symmetry, all the Fourier phases become 0 or $\pi$.

TABLE 4

Herbig Ae/Be Disk Properties

\begin{tabular}{|c|c|c|c|c|}
\hline Target Name & Dust Fraction at $H$ Band $^{\mathrm{a}}$ & $\begin{array}{l}\text { Ring Diameter } \\
\text { (mas) }\end{array}$ & $\begin{array}{l}\text { Ring Diameter } \\
\text { (AU) }\end{array}$ & Comments \\
\hline 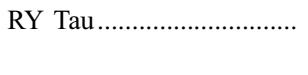 & $0.8 \pm 0.05$ & $\begin{array}{l}3.99 \pm 0.35 \\
2.85 \pm 0.24\end{array}$ & $\begin{array}{l}0.57 \pm 0.05 \\
0.40 \pm 0.04\end{array}$ & $\begin{array}{l}\text { Poor fit with no halo emission } \\
\text { Good fit with } 10 \% \text { halo emission }\end{array}$ \\
\hline  & $0.54 \pm 0.06$ & $3.8 \pm 0.3$ & $0.50 \pm 0.04$ & \\
\hline 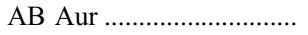 & $0.65 \pm 0.10$ & $3.2 \pm 0.3$ & $0.46 \pm 0.05$ & $7.5 \% \pm 2.5 \%$ halo emission \\
\hline 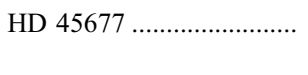 & $\begin{array}{l}0.46 \pm 0.03^{\mathrm{b}} \\
0.62 \pm 0.03^{\mathrm{b}}\end{array}$ & $\begin{array}{l}\sim 15.6 \\
\sim 12.9^{\mathrm{c}}\end{array}$ & $\begin{array}{l}15.6 \\
12.9\end{array}$ & $\begin{array}{c}\text { Strong Asymmetry; see Table } 6 \\
\text { Gaussian FWHM }\end{array}$ \\
\hline 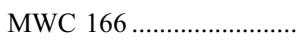 & $0.1 \pm 0.1$ & $10 \pm 5$ & $11.5 \pm 5.8$ & Weak IR excess; nearby companion star \\
\hline  & $0.5 \pm 0.1$ & $4.1 \pm 1.0$ & $0.57 \pm 0.14$ & \\
\hline 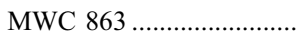 & $0.65 \pm 0.15$ & $4.7_{-0.5}^{+0.8}$ & $0.71_{-0.08}^{+0.12}$ & Poor SED fit \\
\hline 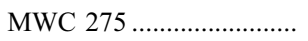 & $0.59 \pm 0.06$ & $3.3 \pm 0.5$ & $0.40 \pm 0.06$ & $5.0 \% \pm 2.5 \%$ halo emission \\
\hline 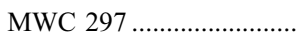 & $0.60 \pm 0.05$ & $5.6 \pm 0.5$ & $1.40 \pm 0.13$ & Possible $\lesssim 5 \%$ halo \\
\hline MWC $614 \ldots \ldots \ldots \ldots \ldots \ldots \ldots \ldots$ & $0.39 \pm 0.03^{\mathrm{d}}$ & $\gtrsim 13$ & $\gtrsim 3.1$ & Suspect nearby companion \\
\hline 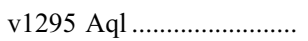 & $0.5 \pm 0.1$ & $3.48 \pm 0.40$ & $1.01 \pm 0.12$ & Assuming $d=290 \mathrm{pc}$ \\
\hline 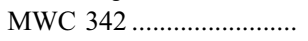 & $0.75 \pm 0.10$ & $3.1 \pm 0.3$ & $3.1 \pm 0.3$ & \\
\hline 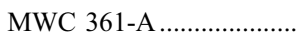 & $0.6 \pm 0.1$ & $3.47 \pm 0.44$ & $1.49 \pm 0.19$ & Primary component only \\
\hline 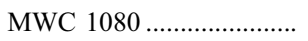 & $0.90 \pm 0.10$ & $3.11 \pm 0.21$ & $6.84 \pm 0.46$ & \\
\hline
\end{tabular}

${ }^{\text {a }}$ Best estimate for fraction of $H$-band light coming from circumstellar material based on most recent photometry. Upper and lower limits are based on SED fitting to diverse data sets and represent the range of possible values given historical variability.

${ }^{\mathrm{b}} H$-band excess derived directly from fitting visibility data. SED analysis for HD 45677 yields estimate for $H$-band dust fraction $0.65 \pm 0.15$.

${ }^{c}$ FWHM result from fitting Gaussian profile instead of ring model.

${ }^{\mathrm{d}} H$-band excess derived directly from fitting visibility data. SED analysis for MWC 614 yields estimate for $H$-band dust fraction $0.37 \pm 0.10$. 


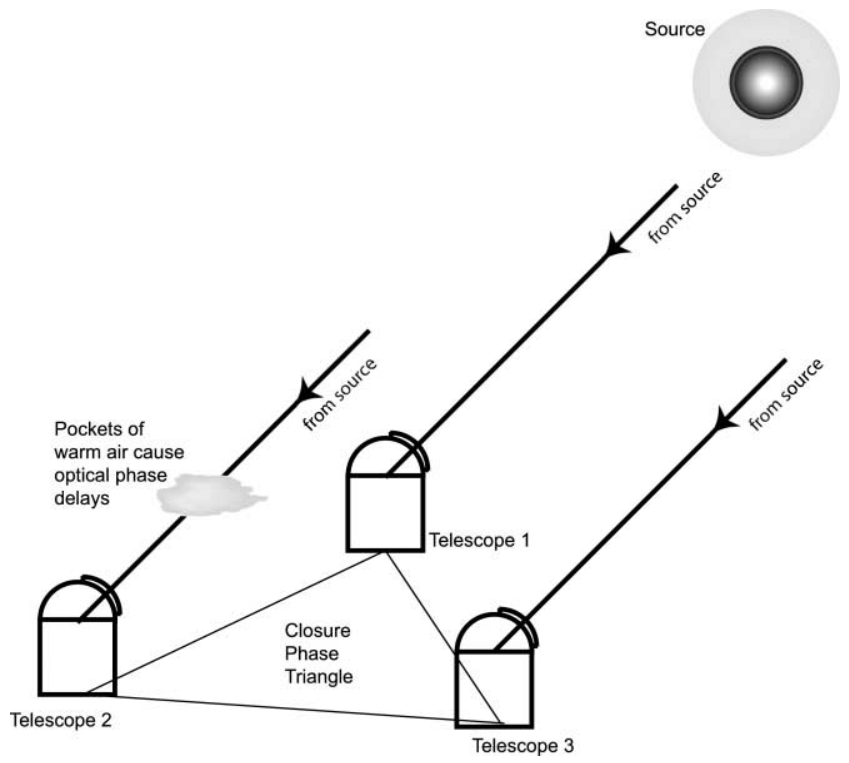

FIG. 19.- Telescope geometry for closure-phase measurement. Turbulent air pockets cause optical path length variations above individual telescopes corrupting fringe phase measurements. However, by summing fringe phases around a closed triangle, these phase errors cancel out, resulting in a good interferometric observable, "the closure phase."

cancels out all atmospheric disturbances within the "closed" triangle, resulting in a good observable, the closure phase, that is independent of the phase fluctuations above the array of telescopes.

The following derivation mathematically expresses the qualitative ideas of the last paragraph. Consider the closure phase $\left(\Phi_{i j k}^{C P}\right)$ for a triangle connecting telescopes $i, j$, and $k$ :

$$
\Phi_{i j k}^{\mathrm{CP}}=\Phi_{i j}^{\mathrm{obs}}+\Phi_{j k}^{\mathrm{obs}}+\Phi_{k i}^{\mathrm{obs}}
$$

where $\Phi_{i j}^{\mathrm{obs}}$ here is the "observed" fringe phase for baseline between telescopes $i$ and $j$. The observed fringe phase can be seen to be equal to the intrinsic phase $\Phi_{i j}$ (this is what we are most interested in) and the difference between the time-varying atmospheric phase delays $\phi_{i}$ associated with individual telescopes. Thus,

$$
\Phi_{i j}^{\mathrm{obs}}=\underbrace{\Phi_{i j}}_{\text {intrinsic }}+\underbrace{\left(\phi_{j}-\phi_{i}\right)}_{\text {atmospheric }} .
$$

Putting these last two equations together we can prove the closure phase to be independent of atmospheric phase delays:

$$
\begin{aligned}
\Phi_{i j k}^{\mathrm{CP}} & =\Phi_{i j}^{\mathrm{obs}}+\Phi_{j k}^{\mathrm{obs}}+\Phi_{k i}^{\mathrm{obs}} \\
& =\Phi_{i j}+\left(\phi_{j}-\phi_{i}\right)+\Phi_{j k}+\left(\phi_{k}-\phi_{j}\right)+\Phi_{k i}+\left(\phi_{i}-\phi_{k}\right) \\
& =\Phi_{i j}+\Phi_{j k}+\Phi_{k i}
\end{aligned}
$$

In principle, images can be reconstructed using only visibilities and closure phases (related to the technique of "self-calibration" in radio interferometry; Cornwell \& Wilkinson 1981) when additional constraints are imposed (such as limited field of view and positivity). A more pedagogical description and derivation of the closure phase can be found in Monnier (2000, 2003).

\subsection{Important Properties of Closure Phases}

The most important property of closure phases follows directly from the properties of the Fourier phases already discussed. That is, a "centrosymmetric" (point-symmetric) image will always have a closure phase of zero degrees. ${ }^{12}$ Thus, detection of a nonzero closure phase is a robust sign of skewed emission, a definitive deviation from point symmetry. This remarkable and robust property of closure phases is key for studies of YSOs, since model-independent image reconstructions are today beyond our capabilities (due to limited $u-v$ coverage).

Another key point is that the interferometer must have sufficient angular resolution to resolve the scale over which the emission is skewed: an unresolved source is always point-symmetric. Thus, one must match interferometer resolution to the appropriate angular scale.

While the detection of skewed emission is robust, quantifying the degree of skew is very model-dependent. When limited $u-v$ coverage forbids direct image reconstruction, one must carry out model fitting. Fortunately, disk theorists routinely produce models for fitting to SEDs, and the results of radiative transfer models are suitable for comparing to interferometry data (e.g., Malbet \& Bertout 1995; D’Alessio et al. 1998; Whitney et al. 2003; Lachaume et al. 2003; Akeson et al. 2005a, Harries et al. 2004).

\subsection{Skewed NIR Disk Emission from YSOs}

In this paper, we compare measured closure phases with physically motivated models for the disk emission in order to quantify the level of skewed emission present in our sample. While future CP surveys with interferometers of $8 \mathrm{~m}$ class telescopes should provide a rich and detailed data set for modeling, we show below that even the limited, first-generation closure phases presented here can distinguish between current competing disk models. Here we contemplate two classes of disk models: the Dullemond-DominikNatta (DDN) disk model and the ad hoc "skewed ring" model.

\subsubsection{DDN Models}

Dullemond et al. (2001) established the current paradigm for Herbig disks (and by extension, even new T Tauri disk models; Muzerolle et al. 2003). As initially outlined by Natta et al. (2001), the star is surrounded by an optically thin cavity with a gas-only disk. The dusty disk begins when the temperature drops below the dust destruction temperature $(T \sim 1500 \mathrm{~K})$, at which point the midplane becomes optically thick. At the dust destruction radius the disk is clearly quite hot, and this "puffs up" the inner rim. The main achievement of Dullemond et al. (2001) was to couple the radiative transfer to a (vertical) hydrostatic disk structure, finding a physically self-consistent height for the inner rim and the outer disk flaring (which match on to traditional flared disk models, e.g., Chiang \& Goldreich 1997).

In the DDN paradigm, the frontally illuminated inner wall is perfectly vertical. These authors comment that such a situation may not be physically reasonable, and indeed a variety of effects may "curve" the inner rim (some of these are discussed in $\S 5.3$ ). In the case of a vertical wall, an observer will generally only see the far side of the inner rim wall, since the front side will be blocked by outer disk material. This scenario produces maximally skewed disk emission, boding well for CP studies of YSOs.

Figure 20 (top left panel) shows a synthetic image for the NIR emission from a DDN disk with a 6 mas inner rim diameter, inclined to our line of sight by $30^{\circ}$. The hot inner wall is assumed to have uniform surface brightness, and we neglect any NIR emission or scattering from the rest of the (cooler) disk. Here we take the half-height of the wall to be $\sim 0.3$ times the inner radius

12 Actually, $180^{\circ}$ is also possible but not relevant for our case of resolved structure around a dominant pointlike star. 

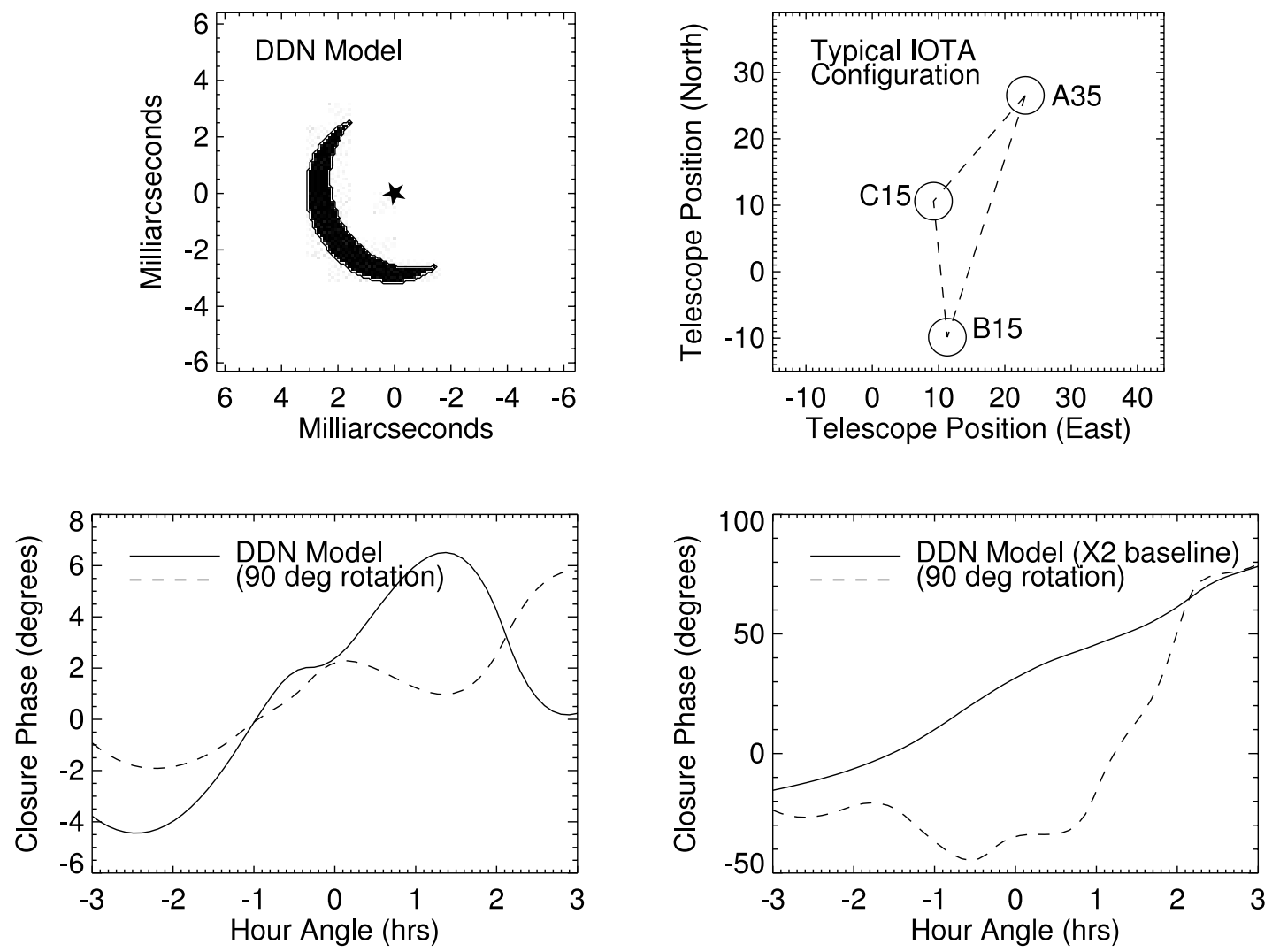

FIG. 20.- Example closure-phase calculation for a DDN disk model using IOTA. The top left panel shows the NIR synthetic image assuming an inner rim radius of 3 mas, viewed at inclination $i=30^{\circ}$. The top right panel shows a typical observing configuration for the IOTA 3-telescope interferometer. The bottom left panel shows the closure phase as a function of hour angle, assuming object at declination $\delta=35^{\circ}$ (dashed line for the DDN model rotated $90^{\circ}$ on the sky). The bottom right panel shows the closure-phase signal for baselines twice as long, illustrating sensitivity with angular resolution.

(following Dullemond et al. 2001). As expected, the disk emission is highly off-center- "skewed" - with respect to the star.

We can calculate the expected CP signal for this disk model using the IOTA interferometer geometry shown in Figure 20 (top right panel), a typical layout used for the observations in this paper. Figure 20 (bottom left panel) show the predicted CP signal for the DDN model (and also a rotated version) for $\lambda=1.65 \mu \mathrm{m}$. Here we also assumed that $65 \%$ of the emission came from the dust, while $35 \%$ arises from the star (typical decomposition for our targets). Figure 20 (bottom right panel) shows how the signal is much stronger if the interferometer has 2 times longer baselinesclearly, the disk appears more skewed with increasing angular resolution.

\subsubsection{Generic Skewed Ring Model}

Later in this paper, we show that the DDN model predicts large closure phases that are not observed. In order to move beyond the vertical wall assumption of the DDN model, we explore the class of skewed ring models. Monnier \& Millan-Gabet (2002) discussed in detail the merits of a ring model for fitting to the near-infrared visibilities of YSOs, and this basic model has been used by most current workers in this field (e.g., MillanGabet et al. 2001; Eisner et al. 2004; Akeson et al. 2005a). The narrow range of temperatures over which dust emits at $1.65 \mu \mathrm{m}$ and the empirical results of Tuthill et al. (2001) imaging of the $\mathrm{LkH} \alpha 101$ disk both suggest that the dust at the inner edge of the YSO accretion disk will appear as a thin ring in the near-infrared. Here we adopt a Gaussian cross section for the ring emission, in order to approximate smoother (i.e., curved) inner rim emission.
We accept this ring model with an additional complicationwe modulate the ring brightness as a function of azimuth by a sinusoid (essentially, an $m=1$ mode). This model was recently used for interpretation of the AB Aur disk by Millan-Gabet et al. (2006c). The top left and right panels of Figure 21 show two different ring models with a skew of 1 and 0.5 (and ring thickness of $25 \%$ using Gaussian profile). The skew refers to the amplitude of the modulation in the ring brightness; thus, a skew of 0 is centrosymmetric, a skew of 0.5 has a $3: 1$ brightness contrast between the brightest and faintest (diametrically opposed) portions of the ring, and a skew of 1 has a contrast formally of $\infty$. Note that the skewed 0.5 image is remarkably similar to the image of LkH $\alpha 101$ reported by Tuthill et al. (2001).

The bottom left and right panels of Figure $21 c$ show the observable closure phases for these models using the same observing geometry introduced for Figure 20. The CP signal for the skew $=1$ model is smaller than the DDN model, as expected since the emission is far less skewed. Also, we see that the skew $=0.5$ model presents even smaller closure phases for the same observing conditions. These calculations clearly illustrate how precision CP measurements of YSOs by today's interferometers can determine the "skewness" of the NIR emission, discriminating between competing disk models (see $\S 5$ ).

If the skewness in disk emission is the result of a disk viewed at intermediate inclination, we might plausibly expect the skewed ring to be elliptical and not circular. This level of detail is beyond the current constraints from the interferometer data and would introduce another free parameter. Thus, we do not consider this reasonable extension to the skewed ring model for the majority of 

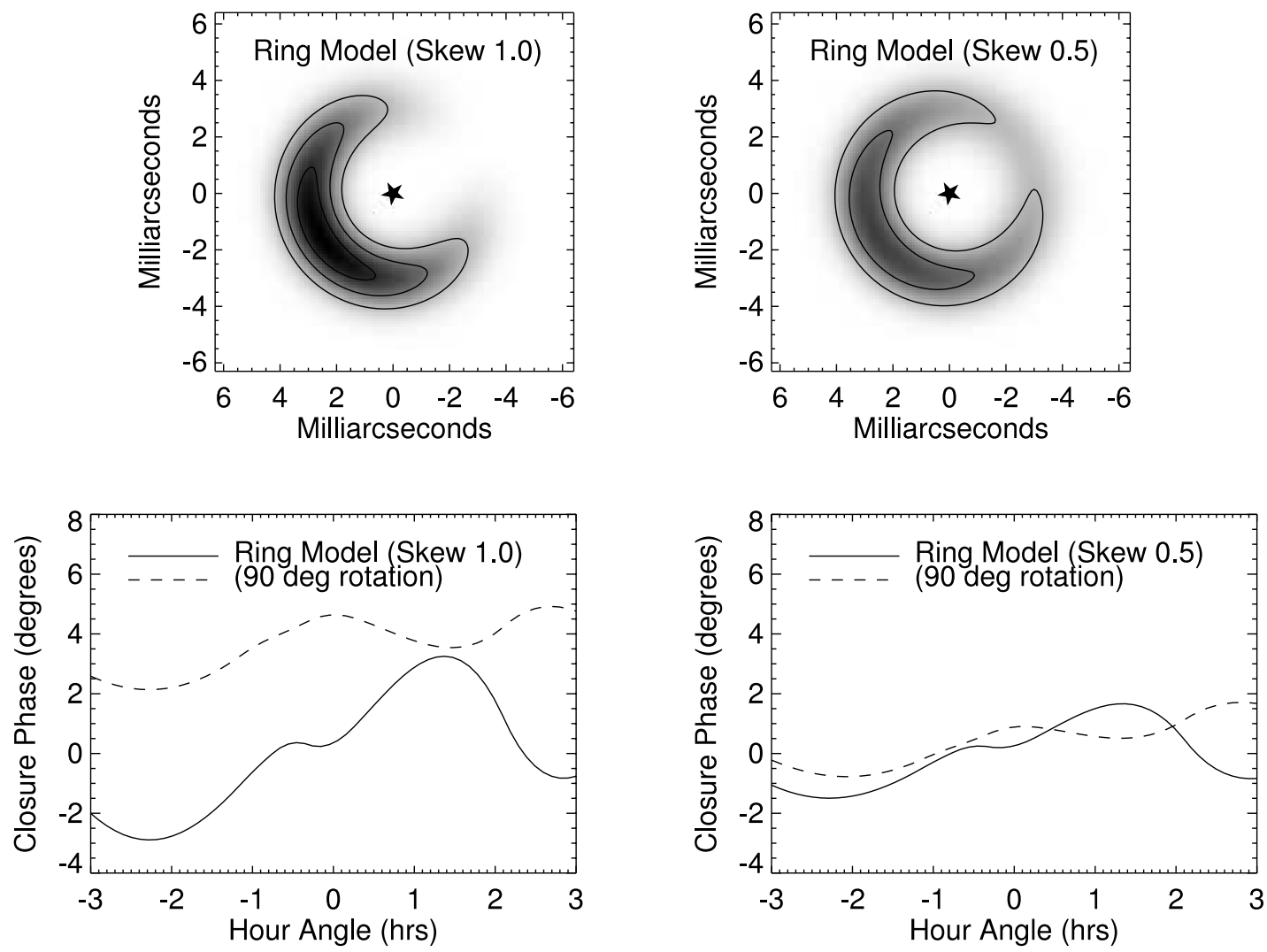

FIG. 21.-Predicted IOTA closure-phase signal for examples of skewed ring models with similar geometry to the DDN model seen in Fig. 20 (see $\S 4.4 .2$ for model details). Top left panel shows synthetic image for ring model with maximum skew $=1$. Top right panel shows synthetic image for ring model with intermediate skew $=$ 0.5. Bottom left panel shows the closure-phase result for skew $=1$ disk (same IOTA observing geometry as in Fig. 20, top right). Bottom right panel corresponds to ring model with skew $=0.5$.

our targets (we do introduce such a sophisticated model for the special case of HD 45677 in $\S 6.1$ ).

\section{ANALYSIS OF CLOSURE PHASES}

Except for AB Aur (Millan-Gabet et al. 2006c), none of our targets have previously been observed using closure phases, and we concentrate our analysis of this section on this observable. We find eight of the 14 sample targets have closure phases consistent with zero within measurement errors. Only AB Aur, HD 45677, MWC 297, MWC 361-A, MWC 614, and MWC 1080 show statistically significant nonzero closure phases (at $2 \sigma$ level).

In order to interpret this result, we must consider the angular resolution of the IOTA interferometer and disk sizes of our targets (measured in $\S 3$ ), since many of our disks are only marginally resolved. Recall from $\S 4$ that closure phases encode deviations from centrosymmetry in an extended object, closely related to the skewness of the emission. Perhaps best explained in Lachaume (2003), marginally resolved objects naturally possess small closure phases even if the inherent emission is extremely asymmetric. An intuitive explanation of this is to consider that any "unresolved" source must be considered to be centrosymmetric.

Thus, our first priority is to determine whether the finding of small closure phases reflects a true lack of skewness in the YSO disk emission or rather results from inadequate angular resolution. To further this investigation, we have collected the maximum closure phase observed for each target in Table 5, along with the disk size measured in $\S 3$ in units of the fringe spacing $(\lambda / B)$ of the longest IOTA baseline in the appropriate triangle. We confront the specific predictions of the DDN model in the next section.

\subsection{Confronting DDN Models}

In order to extract quantitative information from the near-zero closure phases of most stars in our sample, we first explore the CP predictions of DDN (Dullemond et al. 2001) models described in $\S$ 4.4.1. In order to explore parameter space, we generated CP predictions for DDN models with a range of inner rim diameters

TABLE 5

Closure-Phase Results

\begin{tabular}{|c|c|c|c|}
\hline Target & $\begin{array}{c}\text { Maximum } \\
\text { Closure Phase }^{\mathrm{a}} \\
\text { (deg) }\end{array}$ & $\begin{array}{c}\text { Minimum Fringe } \\
\text { Spacing } \\
\text { (mas) }\end{array}$ & $\begin{array}{l}\text { Ring Diameter } \\
\text { (fringes) }\end{array}$ \\
\hline RY Tau. & $0.1 \pm 0.4$ & 11.5 & 0.35 \\
\hline MWC $480 \ldots \ldots \ldots . . .$. & $0.1 \pm 2.5$ & 8.9 & 0.43 \\
\hline 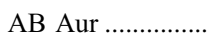 & $-4.1 \pm 0.4^{*}$ & 11.7 & 0.27 \\
\hline HD $45677 \ldots \ldots \ldots . . .$. & $-26.6 \pm 4.0^{*}$ & 11.4 & 1.37 \\
\hline MWC $166 \ldots \ldots \ldots . .$. & $1.8 \pm 0.9$ & 12.1 & 0.83 \\
\hline HD $144432 \ldots \ldots \ldots$ & $-1.6 \pm 1.3$ & 13.5 & 0.30 \\
\hline MWC $863 \ldots \ldots \ldots . . .$. & $5.1 \pm 2.6$ & 11.6 & 0.41 \\
\hline MWC $275 \ldots \ldots \ldots . .$. & $-0.6 \pm 0.4$ & 11.8 & 0.28 \\
\hline MWC $297 \ldots \ldots \ldots . . .$. & $-1.9 \pm 0.4^{*}$ & 9.6 & 0.58 \\
\hline MWC 614 .............. & $4.1 \pm 1.7^{*}$ & 9.3 & $>1.4$ \\
\hline v1295 Aql ............... & $1.9 \pm 1.7$ & 9.4 & 0.37 \\
\hline MWC $342 \ldots \ldots \ldots . . .$. & $-1.8 \pm 1.6$ & 8.9 & 0.35 \\
\hline MWC 361-A ........ & $31.0 \pm 1.5^{*}$ & 10.2 & Binary \\
\hline MWC $1080 \ldots \ldots \ldots$ & $-1.3 \pm 0.5^{*}$ & 9.8 & 0.32 \\
\hline
\end{tabular}

Note.-An asterisk indicates greater than $2 \sigma$ detection.

${ }^{a}$ This column contains the most statistically significant $\mathrm{CP}$ data showing a deviation from zero, based on $u-v$ averaged data. 




FIG. 22.- Comparison of the maximum predicted closure phases for DDN models as a function of disk inclination angle and angular resolution of interferometer. For well-resolved disks, the closure-phase signals for DDN models are expected to be huge but are not observed in any targets of our sample. A few of the most notable targets are labeled.

and viewing angles. In each case, we assumed the disk made up $65 \%$ of the emission at $H$ band with the remaining $35 \%$ arising from the (unresolved) star, a typical case for our target sample. We then generated synthetic closure phases for the actual observing triangles used by IOTA. The main results of this parameter study are shown in Figure 22.

In Figure 22, we plot the maximum observable IOTA closure phase for DDN disks for three different inclinations as a function of the ring diameter. The ring diameter is expressed here in units of the fringe spacing $\lambda / B$ of the longest baseline in a given IOTA closure triangle. For each skewed ring we sampled all possible P.A.s on the sky and here plot the maximum value of the $\mathrm{CP}$ magnitude. While we typically do not know the sky orientation of a given object, we expect our collection of 14 targets to sample random position angles on the sky.

This procedure quantifies the qualitative results from the DDN examples in Figure 20, that well-resolved DDN disks have large $\mathrm{CP}$ signals. In Figure 22, we can then compare the synthetic curves to the maximum closure phases actually observed in our survey. To do so only requires us to account for the differing sizes of each target from $\S 3$. We exclude MWC 361-A from this analysis since the large closure phases for this target likely come from the binary companion, not skewness in the disk around the primary star.

On the basis of this comparison of DDN model predictions with our CP data set, we can draw two primary conclusions.

1. The most well-resolved disk sources have smaller closure phases than the DDN model calculations, although usually nonzero. This indicates some modest skewness in the disk emission but is incompatible with the large skew signal predicted by DDN models.

2. When the ring diameter is less than the $\sim \frac{1}{2}$ fringe spacing of the longest baseline in a triangle, the CP signal is strongly suppressed. This applies to approximately half of our target sample. We require approximately $2-3$ times better angular resolution to fully characterize disk skewness in our sample (corresponding to $70-100 \mathrm{~m}$ baselines at $H$ band or $100-150 \mathrm{~m}$ baselines at $K$ band).

For any given target, the putative disk skewness might be orientated along a position angle not sampled by the IOTA interferometer, which is most sensitive to skew in the north-south

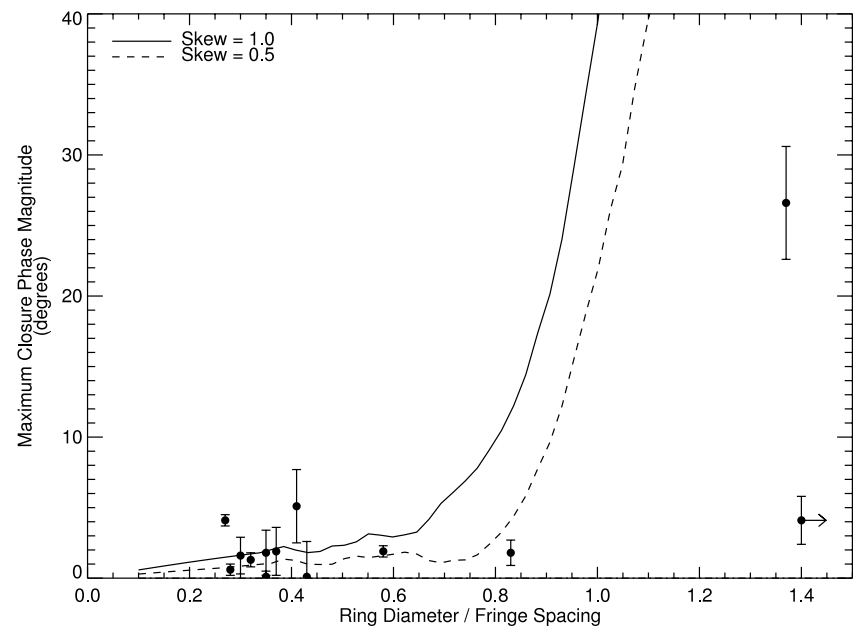

FIG. 23.- Comparison of the maximum predicted closure phases for skewed ring models as a function of the skew amount and angular resolution of interferometer. Note that the line for skew $=0$ is identically zero due to centrosymmetry. Note that most systems are not resolved enough to constrain the skewed models, calling for longer-baseline interferometers such as PTI, CHARA, and VLTI.

direction (due to nonuniform $u-v$ coverage). Thus, even highly skewed disk emission can be "hidden" from the interferometer due to unfavorable observing geometry. However, the small observable closure phases for the most resolved disks (MWC 297, MWC 166, MWC 614, MWC 480, and RY Tau), when taken as a whole, argue strongly against the vertical wall of the inner rim put forward in the DDN model, since this geometry always predicts strong skew in disks viewed from all inclination angles. Note that the DDN model (with vertical inner wall) also predicts highly "elongated" disk emission (see Fig. 20) even for nearly face-on disks, inconsistent with the $\mathcal{V}^{2}$ results of Eisner et al. (2004). Note that AB Aur uniquely appears to show definite skewed emission in its halo (beyond the inner rim), further discussed in Millan-Gabet et al. (2006c).

\subsection{Confronting Skewed Ring Models}

The analysis of the last section was also carried out using the skewed ring model described in $\S$ 4.4.2. The results of this study, along with the observed closure phases, are plotted in Figure 23. While skew $=1$ disks do generally overpredict the CP magnitude, our data cannot clearly distinguish between the models with varying skew factors. Although ad hoc, the skewed ring models are physically motivated and can currently explain the observed visibility and CP data in our sample, including the imaging data for $\mathrm{LkH} \alpha 101$ and the CP data of HD 45677 (taking ellipticity into account; see $\S 6.1$ ). While it is critical to develop "physical" models for the disk emission, the introduction of the skewed ring model offers a new and useful tool for describing the emission independent of the underlying physical models.

For some disks, it should be possible to do more detailed model fitting by including inclination and position angle information known from $\mathrm{CO}$ data or scattered light images (e.g., AB Aur, MWC 275). In these cases, the IOTA closure phases can play a more definitive role in testing specific models. However, in general, longer baseline closure phases and more uniform Fourier coverage will be needed to refine future models of the inner rim geometry.

\subsection{Discussion}

\subsubsection{Implications on Inner Rim Geometry}

The most significant result from this paper is the report of few skewed disks around Herbig Ae/Be stars. This is robustly shown 
by the uniformly small closure phases found in our sample of YSOs. When the sample is viewed as a whole, the small closure phases have been shown to be incompatible with generic predictions of the original DDN disk models; these models possess vertical walls at the inner edge of the accretion disk introducing strongly skewed emission at all viewing angles. Note that this result does not invalidate the overall success and applicability of the DDN model, which mostly focuses on other aspects of the disk structure. While we have shown that skewed ring models are better suited for explaining the small observed closure phases, we also demonstrate that detailed determinations of skew require longer baselines than possible with IOTA.

In the context of today's disk models, our data lend support to inner disk models like that of Isella \& Natta (2005), which includes a "curved" inner rim. In this case, the inner rim curves away from the midplane due to the expected pressure dependence of the dust evaporation temperature. We plan to explore quantitatively the expected $\mathrm{CP}$ signals of this model with future radiative transfer work, but this is beyond the scope of this paper.

Note that there are other mechanisms that could cause the inner rim to curve. One new idea we suggest is a consequence of dust settling and growth, which leads to larger dust grains in the midplane than in the upper layers of the disk. Since large grains better radiate in the infrared, they are able to exist closer to the star. Smaller grains in the upper layers have difficulty cooling and thus evaporate more easily. Thus, the inner rim can be highly curved if the average dust size varies significantly as a function of disk scale height in the inner rim. This process can cause a much greater curvature in the inner rim compared to the effects of gas pressure on the evaporation temperature. Indeed, other recent works have found evidence for dust settling and growth in YSO disks (e.g., Rettig et al. 2005; Duchêne et al. 2004), and the implications on the inner rim structure have only just begun to be explored (e.g., Tannirkulam et al. 2005).

Understanding the geometry of the inner disk is not just important for understanding the NIR emission. Van Boekel et al. (2005) showed that $20 \%-30 \%$ of the mid-IR emission can come directly from the inner rim. Given the intriguing changes in the silicate feature observed using the MIDI instrument on VLTI (van Boekel et al. 2004), it is critical to understand how the dust properties of the rim might be different than those in the surface layers of the disk. In addition, high spectral resolution $\mathrm{CO}$ observations of NIR fundamental and first-overtone lines (Najita et al. 2003; Brittain et al. 2003; Blake \& Boogert 2004) require temperature and density modeling of the inner AU of Herbig disks. Currently, these models have not incorporated the puffed inner wall into their calculations, an improvement urgently needed in order to correctly interpret the potentially powerful kinematic and temperature information available.

\subsubsection{The Halo Phenomenon}

By using three telescopes, our survey has measured visibilities on a larger range of scales than previous work using only two telescope interferometers. We find evidence for intermediate- to large-scale halos around AB Aur, MWC 275, MWC 297, and RY Tau. For our purposes here, we define halo emission to be any extended emission on scales between 10 and 500 mas making up $5 \%-20 \%$ of the NIR flux. Similar extended halos have been reported previously (albeit on 0."5-2."0 scales) around some T Tauris and Herbigs using infrared speckle interferometry (Leinert et al. 1993, 2001) and even on smaller scales probed by lunar occultation (e.g., case of DG Tau; Leinert et al. 1991; Chen et al. 1992). Similarly sized extended emission has recently been reported around a sample of FU Ori objects using the Keck Interferometer (Millan-Gabet et al. 2006b).

The existence of these halos is not well understood theoretically but could arise from diffuse scattered light at large (5$100 \mathrm{AU}$ ) radii (as recently discussed by Akeson et al. 2005b) from the central star, or even from localized thermal emission within a few AU. The origin of the halo material is unclear but plausibly could be from an infalling remnant envelope, dust entrained in the stellar wind/outflow, or (in the case of FU Ori) ejected gas and dust from an earlier outburst. The connection to the hybrid halo+disk model of Vinković et al. (2006) is ambiguous, and our findings should motivate further work on such multicomponent models. In the special case of AB Aur (Millan-Gabet et al. 2006c), we detected nonzero closure phases from the halo component, leading us to conclude that the source of the emission was localized and within 1-4 AU of the star. For the other halos reported in this paper, we can only roughly constrain the location of the emission (10-500 mas); further observations with short baselines will be required to elucidate the nature of halos around Herbig Ae/Be stars.

Recently, Baines et al. (2006) report interesting "spectroastrometry" observations of many Herbig stars, finding evidence for possible binaries around many targets (including AB Aur, HD 45677, v1295 Aql, and MWC 361-A of this sample). While we caution that a few of our halo detections may turn out to be due to very close binary companions (10-500 mas), most stars in our sample have been surveyed for companions using adaptive optics down to about 100 mas (e.g., AB Aur, MWC 297, v1295 Aql; Eisner et al. 2004). Similarly, the spectro-astrometry data can misinterpret halo emission as evidence for binarity if the extended emission itself is skewed (as has been shown for AB Aur; MillanGabet et al. 2006c). Note that scattered disk/halo light can only affect spectro-astrometry results if it has a substantially different $\mathrm{H} \alpha$ spectrum than what we see from the star. This could happen perhaps in two ways. The $\mathrm{H} \alpha$ emission from the star is probably not uniformly distributed but more concentrated near polar regions and will also have a different dynamical signature/line profilethus, the observer will see different $\mathrm{H} \alpha$ than the disk midplane, which then scatters the light into our line of sight. Second, stellar light must traverse long paths just above the disk midplane before scattering into our line of sight; thus, there could be significant $\mathrm{H} \alpha$ absorption (depending on ionization state). These effects deserve more study before settling on an interpretation of the spectroastrometry data.

\section{SPECIAL TREATMENT FOR HD 45677 AND MWC 361}

\subsection{Parametric Imaging of the HD 45677 Disk}

One exception to the general pattern of small closure phases is HD 45677, which is both heavily resolved, elongated, and shows strong closure phases. We already fit a symmetric ring and Gaussian to the model in $\S 3$ and Figure 8 and noted the large fit residuals due to elongated and skewed structures evident in the $\mathcal{V}^{2}$ and CP data.

First we fit the $\mathcal{V}^{2}$ data with an elongated ring model; the results of this fit are shown in the top panels of Figure 24. The direction of elongation (P.A. $70^{\circ}$ ) is similar to the observed (visible) polarization angle of $60^{\circ}-80^{\circ}$ (Coyne \& Vrba 1976). However, as expected, this model cannot explain the relatively large observed closure phases that must arise from skewed emission. The results of this fit are given in Table 6.

In order to explore this, we allowed the elongated ring to be skewed, following the same method as for the skewed ring model in $\S$ 4.4.2. That is, the elongated ring emission is modulated as a 

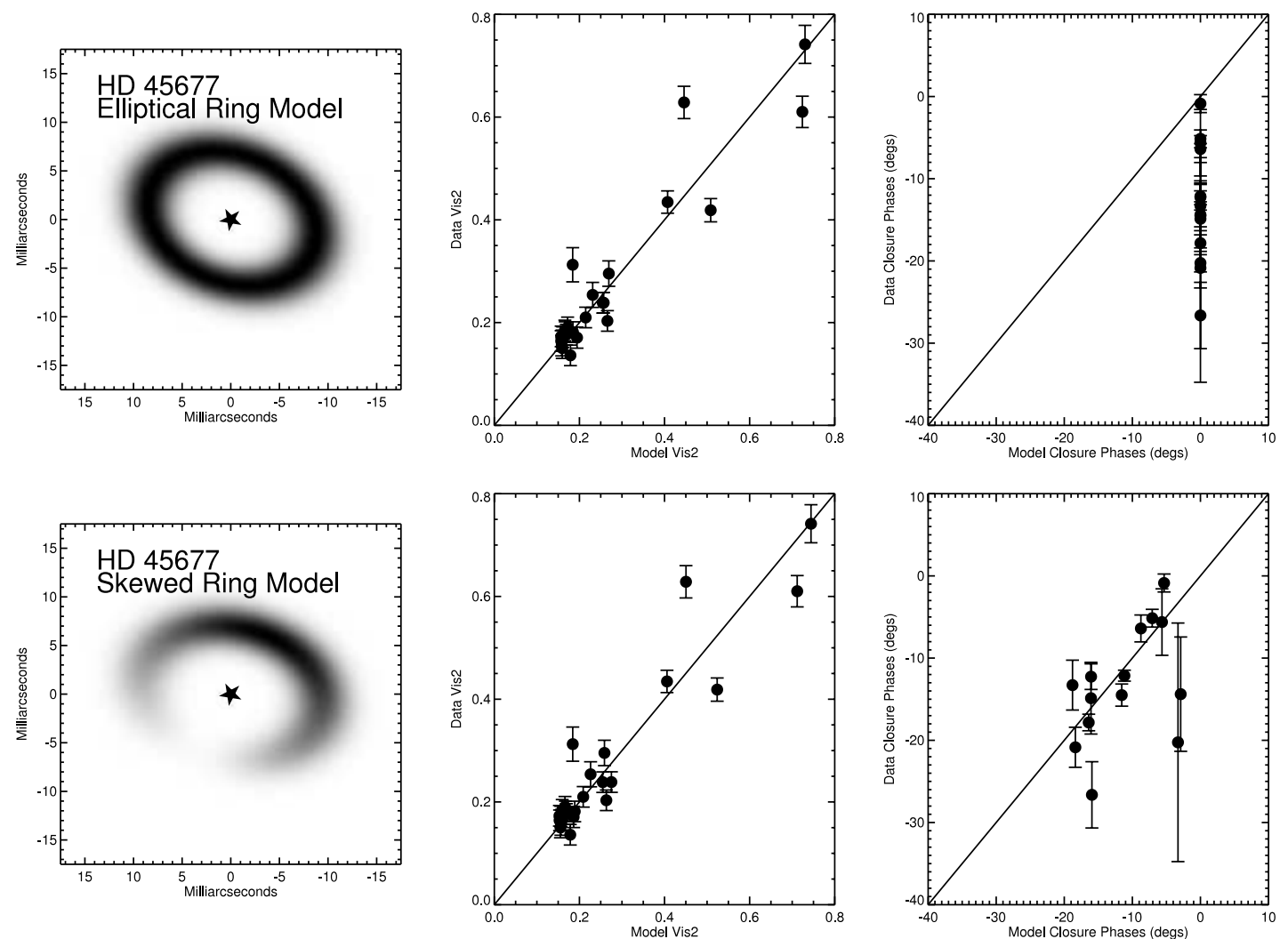

FIG. 24.- -Top left panel shows the best-fit elliptical ring model to the IOTA3 data (north is up and east is left). Top middle panel compares the observed visibility data with the model data. Top right panel compares the observed closure phases with the model closure phases. Since this model is constrained to be centrosymmetric, all model closure phases are identically zero and thus a good fit is impossible. The bottom panels present the best-fit "skewed elliptical ring model." The closure phases are fairly well explained by a strong northwest skew. All parameters of these fits are presented in Table 6. Some data points are not well explained by these models, suggesting a patchier dust distribution.

function of azimuth. The best-fit parameters are found in Table 6 , and the synthetic image is shown in Figure 24 (bottom panels). Interestingly, the skew angle (position angle from central star with greatest skewed brightness) is $\sim 90^{\circ}$ different than the major axis of the elongated ring, a geometry that might be expected for a flared disk (e.g., Malbet et al. 2001). Given our parametric image and the direction of the skew, we would predict that scattering by dust would produce a net linear polarization of P.A. $\sim 70^{\circ}$, in agreement with observations. The $\chi^{2}$ fit to the $\mathcal{V}^{2}$ is poor at the shortest baselines, suggesting additional complexity missing from our model.

The skewed ring model presented here can be viewed as the first attempt to do "parametric" imaging of YSO disks using long baseline interferometry. While the number of degrees of freedom in our model is still relatively small, we are reconstructing fairly complicated image characteristics. Similar quality "images" should be possible for the rest of our target sample in the coming years.

Lastly, we want to comment that this ring image is qualitatively similar to the expected results outlined in previous papers (Coyne \& Vrba 1976; Oudmaijer \& Drew 1999). Based on the relatively cool infrared excess and the linear polarization angle, a ringlike, elongated morphology was predicted and the position angles found here are entirely consistent with the polarization signatures. Combining infrared polarimetry with interferometry could reveal unique aspects of the dust in this system (e.g., Ireland et al. 2005).

We believe that our data support the idea that HD 45677 is not a bona fide young stellar object, but rather a more evolved system akin to CI Cam (e.g., Miroshnichenko et al. 2002). The disk size is too large to be a typical YSO system (unless we are observing a unique transition object). Perhaps a binary interaction in the recent past created the disklike dust distribution around this source (e.g., Miroshnichenko et al. 2005 and references therein). Studies of this dust shell may shed light on the mysterious process of dust production around some main-sequence Be stars.

\subsection{Astrometric Orbit for the Young Binary MWC 361-A}

The first definitive detection of the MWC 361-A close binary was reported by Millan-Gabet et al. (2001) using interferometry.

TABLE 6

Elongated Ring Models for HD 45677

\begin{tabular}{|c|c|c|c|c|c|c|c|}
\hline \multirow[b]{2}{*}{ Model Type } & \multirow[b]{2}{*}{ Dust Fraction at $H$ band } & \multirow[b]{2}{*}{ Star Fraction at $H$ band } & \multicolumn{3}{|c|}{ Ring Diameter } & \multirow[b]{2}{*}{ SKEW } & \multirow[b]{2}{*}{ SKEW P.A. } \\
\hline & & & Major & Minor & P.A. & & \\
\hline  & $0.46 \pm 0.01$ & $0.54 \pm 0.01$ & $17.8 \pm 1.3$ & $12.9 \pm 1.4$ & $70 \pm 16$ & 0 & $\cdots$ \\
\hline Skewed elliptical ring ................ & $0.46 \pm 0.01$ & $0.54 \pm 0.01$ & $18.5 \pm 1.5$ & $13.4 \pm 0.6$ & $77 \pm 13$ & $0.92 \pm 0.08$ & $-31 \pm 8$ \\
\hline
\end{tabular}


TABLE 7

Astrometric Binary Solutions for MWC 361-A

\begin{tabular}{|c|c|c|c|c|}
\hline $\begin{array}{l}\text { Epoch } \\
\text { (UT) }\end{array}$ & $\begin{array}{c}\Delta \alpha \\
\text { (mas) }\end{array}$ & $\begin{array}{c}\Delta \delta \\
\text { (mas) }\end{array}$ & $\begin{array}{l}\text { Separation } \\
\text { (mas) }\end{array}$ & $\begin{array}{l}\text { P.A. } \\
\text { (deg) }\end{array}$ \\
\hline 1998 Jun $13-21$. & $0.9 \pm 3.0$ & $17.9 \pm 2.6$ & 17.9 & 2.7 \\
\hline 1998 Sep 29. & $2.9 \pm 3.1$ & $17.7 \pm 1.1$ & 18.0 & 9.4 \\
\hline 2003 Jun $21-22$. & $11.4 \pm 4.6$ & $3.5 \pm 2.9$ & 11.9 & 73.0 \\
\hline 2003 Nov $27-29$. & $5.8 \pm 3.8$ & $-5.8 \pm 1.0$ & 8.2 & 134.8 \\
\hline 2004 Jun 6 & $1.7 \pm 2.6$ & $-11.3 \pm 1.2$ & 11.4 & 171.6 \\
\hline 2004 Dec 10 & $-3.3 \pm 3.4$ & $-1.9 \pm 0.6$ & 3.9 & 239.8 \\
\hline 2005 Jun $17-29 \ldots \ldots \ldots \ldots$ & $-2.6 \pm 2.0$ & $12.5 \pm 0.8$ & 12.8 & 348.3 \\
\hline
\end{tabular}

Note.-Other binary information: $H$-band ratio: $6.5 \pm 0.5$. Primary UD diameter: $3.6 \pm 0.5$ mas.

As previously already mentioned, spectroscopic evidence has also been accumulating, and Pogodin et al. (2004) recently reported a preliminary single-lined radial velocity $(\mathrm{RV})$ orbit of the primary with a period of 1341 days.

There are few simultaneous radial velocity and astrometric orbits for young star binaries (e.g., HD 98800-B; Boden et al. 2005). ${ }^{13}$ These data are particular valuable because they directly constrain the stellar masses and sometimes the system distance, invaluable "ground truth" tests for theories of stellar interiors and evolution.

We report five new epochs of $\mathcal{V}^{2}$ and CP data for MWC 361-A in this paper. All these epochs have been fitted for a binary system, with the additional complication that the primary is clearly partially resolved. For the treatment in this paper, we have assumed the fainter secondary star is completely unresolved, as expected for the size-luminosity relations of Herbig stars. We also have independently refit the two epochs of data from MillanGabet et al. (2001) in order to extend our time coverage over $\sim 5$ years, more than 1.5 orbital periods.

Table 7 shows our best-fit positions for the secondary component relative to the primary for each of the seven epochs. Furthermore, our fitting found a brightness ratio of $6.5 \pm 0.5$ (primary/ secondary) at $H$ band and a primary uniform disk diameter of $3.6 \pm 0.5$ mas (we analyzed the primary disk size in $\S 3$ and Fig. 17). There was some evidence for variations in the flux ratio with time, as expected for young stars and previously observed for the Z CMa system (e.g., Millan-Gabet \& Monnier 2002). For about half the epochs (1998 June, 2003 November, 2004 June, and 2005 June) we were able to uniquely constrain all the free parameters. For the remaining, we had to fix the brightness ratio and size of the components to yield a good position (due to more limited data sets). In a few cases, we had to use a priori information concerning the likely binary position to break position degeneracies. For instance, the 1998 September data come from only one night, and multiple solutions exist; however, only one solution is sufficiently close to the unambiguous result of 1998 June to be considered plausible and consistent with the other epochs.

Figure 25 shows error ellipses for the position of the secondary relative to the primary for all epochs. The position predictions for our best-fit orbit are also shown and the agreement is excellent. The orbital parameters (and conservative error estimates) are contained in Table 8 and compared to the orbital elements of Pogodin et al. (2004). All orbital elements agree within observational uncertainties. A future paper will fit the

\footnotetext{
13 Eclipsing binary systems are also powerful tools for determining stellar masses (e.g., Stassun et al. 2004).
}

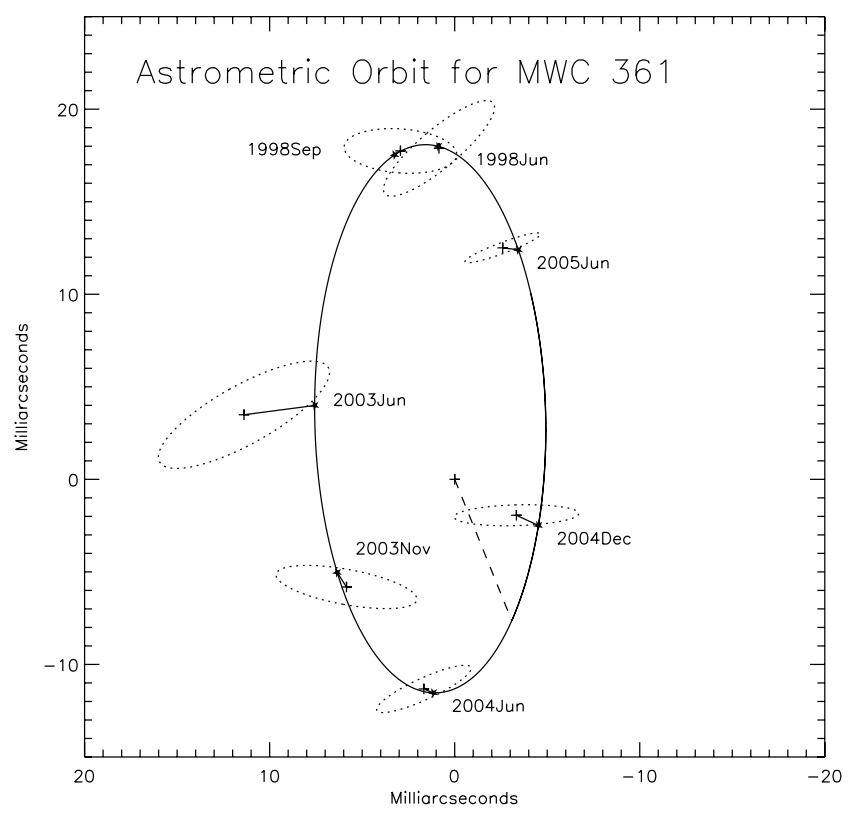

FIG. 25.-Astrometric orbit for MWC 361-A (solid line). The observed locations of the secondary component (relative to primary) are marked with error ellipses (dotted lines with crosses) for seven different epochs. The dashed line connects the locations of the primary and secondary star at periastron passage.

$\mathcal{V}^{2}$ and CP data directly (along with radial velocity data) using the orbital elements (as recently done using IOTA data for $\lambda$ Vir by M. Zhao et al. 2006, in preparation). This analysis will yield a joint orbital solution with significantly smaller parameter errors; however, this analysis is beyond the scope of this paper.

With the combined RV and astrometric orbital solution, we can determine physical characteristics of the MWC 361-A system. We had the Hipparcos intermediate data reanalyzed using the new orbital parameters in an attempt to improve the parallax and constrain the photocenter orbit (in the method of Pourbaix $\&$ Jorissen 2000). The new result $\pi=2.76 \pm 0.68$ mas $(d=$ $\left.360_{-70}^{+120} \mathrm{pc}\right)$ varies little from the original catalog result $(\pi=$ $2.33 \pm 0.62$ mas; $d=430_{-90}^{+160} \mathrm{pc}$ ). Using the orbital period ( $3.74 \pm 0.06 \mathrm{yr}$, the weighted average between the independent $\mathrm{RV}$ and astrometric determinations) and (angular) semimajor axis (15.14 \pm 0.70 mas), we can calculate the total system mass $M_{1}+M_{2}=10.4_{-5.9}^{+20.5}$ using the original Hipparcos measurement

TABLE 8

Preliminary Orbital Parameters for MWC 361-A

\begin{tabular}{|c|c|c|}
\hline Parameter & $\begin{array}{c}\text { Radial Velocity } \\
\text { (Pogodin et al. 2004) }\end{array}$ & $\begin{array}{l}\text { Interferometry } \\
\text { (This Work) }\end{array}$ \\
\hline Period (days) .................. & $1341 \pm 41$ & $1377 \pm 25$ \\
\hline$T_{0}(\mathrm{JD}) \ldots \ldots \ldots \ldots \ldots \ldots \ldots \ldots \ldots \ldots \ldots \ldots$ & $2449149 \pm 87$ & $2449152 \pm 90$ \\
\hline$a_{1} \sin i(\mathrm{AU}) \ldots \ldots \ldots \ldots \ldots$ & $1.33 \pm 0.08$ & $\ldots$ \\
\hline$a$ (mas) & $\ldots$ & $15.14 \pm 0.70$ \\
\hline e & $0.29 \pm 0.07$ & $0.30 \pm 0.06$ \\
\hline 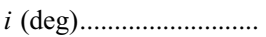 & $\ldots$ & $65 \pm 8$ \\
\hline$\omega(\operatorname{deg})$ & $203 \pm 22$ & $224 \pm 16$ \\
\hline$\Omega(\operatorname{deg}) \ldots \ldots \ldots \ldots \ldots \ldots \ldots$ & $\ldots$ & $-0.2 \pm 7.6$ \\
\hline$K\left(\mathrm{~km} \mathrm{~s}^{-1}\right) \ldots \ldots \ldots \ldots \ldots \ldots$ & $11.2 \pm 0.7$ & $\ldots$ \\
\hline$f\left(M_{2}\right)\left(M_{\odot}\right) \ldots \ldots \ldots \ldots \ldots$ & $0.175 \pm 0.035$ & $\ldots$ \\
\hline$M_{1}+M_{2}\left(M_{\odot}\right)^{\mathrm{a}} \ldots \ldots \ldots \ldots$ & $10.4_{-5.9}^{+20.5}$ & $10.4_{-5.9}^{+20.5}$ \\
\hline
\end{tabular}

${ }^{\text {a }}$ For distance $d=430_{-90}^{+160} \mathrm{pc}$; see description of system mass estimate in $\S 6.2$. 
(these errors represent $1 \sigma$ confidence intervals using a Monte Carlo method for error propagation). Unfortunately, while these preliminary masses are consistent with our expectations for a binary system of early B stars, the large distance errors propagate into large mass uncertainties, rendering these estimates useless for constraining any models. It will be crucial to extract a "doublelined" radial velocity orbit in order to determine an "orbital" parallax, resulting in accurate and precise mass estimates for both components.

We note that Pogodin et al. (2004) measured the mass function $f\left(M_{2}\right)$ for the secondary from the single RV orbit. This result $\left[f\left(M_{2}\right)=0.175 \pm 0.035\right]$ was interpreted using an assumed inclination $i=70^{\circ}$, consistent with our measurement of $i=$ $65^{\circ} \pm 8^{\circ}$. Thus, we can concur with their analysis that MWC 361-A appears to be a close binary consisting of an early Be star, likely hosting the resolved disk of $\S 3$, and a lower mass, late $\mathrm{Be}$ star as secondary.

The periastron distance of the MWC 361-A components is only $\sim 4 \mathrm{AU}$ (due to somewhat eccentric $e \sim 0.3$ orbit); thus, the accretion disk around the MWC 361-A primary should be severely truncated (perhaps this relates to recent difficulty in fitting the far-infrared SED; Elia et al. 2004). Certainly, MWC 361-A provides a fascinating laboratory for studying binary star formation and is suitable for future studies by longer baseline interferometers that can both measure orbital parameters at high accuracy but also image the accretion disks around both stellar components. It is extremely rare to find young stars of such early spectral types in a binary system, and MWC 361-A promises to be a new anchor point for models of stellar interiors and evolution, as well as for disk evolution in binary systems.

\section{CONCLUSIONS}

Here we have presented the results of the first closure-phase survey of young stellar objects using an infrared interferometer. We find that most YSO targets show no signs of skewness in their emission on the scale of $\sim 4$ mas. Our data are incompatible with vertical wall models of the puffed-up inner rim (e.g., Dullemond et al. 2001). A skewed ring model was developed in order to quantify our results, and the observed small closure phases favor inner dust rims that are smoothly curved (e.g., Isella \& Natta 2005). At least a crude understanding of the inner AU of YSO disks is crucial for interpreting a host of other YSO observations, including spectrally resolved CO data, spectro-astrometry, mid-IR sizes, and spatially resolved silicate feature minerology. Baselines significantly longer than the maximum IOTA length of $38 \mathrm{~m}$ (i.e., from CHARA and VLTI) will be required to investigate this further for specific sources.
An independent and complete reanalysis of new $H$-band $\mathcal{V}^{2}$ data found fresh evidence for extended halos of emission with about $5 \%-10 \%$ of the emission for a few targets (see also Leinert et al. 2001). Halos appear to be quite common in many young disk systems ( T Tauri, Herbig, FU Ori), although the physical origin of this large-scale emission appears not yet understood. These new data may reopen the debate concerning the relative importance of disks, halos, envelopes, localized thermal emission, and the applicability of hybrid models.

We also took the first steps toward imaging young stellar objects by studying the unusual system HD 45677, finding this Be star to be surrounded by an elongated and highly skewed dust ring. We agree with earlier suggestions that HD 45677 may not be a young object, but rather a member of the enigmatic class of main-sequence hot stars with large IR excess.

Lastly, we calculated the first astrometric orbit for the shortperiod binary MWC 361-A, finding good agreement with recent spectroscopic work. We found mass estimates in line with expectations and discussed the exciting science potential from future studies of this unique system.

J. D. M. thanks D. Pourbaix for reanalyzing the Hipparcos parallax of MWC 361-A using the new orbital parameters and A. Tannirkulam for photometry from the MDM Observatory. The authors gratefully acknowledge support from the Smithsonian Astrophysical Observatory, NASA (for third telescope development and NASA NNG05G1180G), the National Science Foundation (AST 01-38303, AST 03-52723), and the Jet Propulsion Laboratory (JPL awards 1236050 and 1248252). E. P. was partially supported by a SAO Predoctoral fellowship, J. D. M. by a Harvard-Smithsonian CfA fellowship, and R. M.-G. and J.-P. B. were partially supported through NASA Michelson Postdoctoral Fellowships. The IONIC3 instrument has been developed by LAOG and LETI in the context of the IONIC collaboration (LAOG, IMEP, LETI). The IONIC project is funded by the CNRS (France) and CNES (France). This research has made use of the SIMBAD database, operated at CDS, Strasbourg, France, and NASA's Astrophysics Data System Abstract Service. This publication makes use of data products from the Two Micron All Sky Survey, which is a joint project of the University of Massachusetts and the Infrared Processing and Analysis Center/California Institute of Technology, funded by the National Aeronautics and Space Administration and the National Science Foundation. This work has made use of services produced by the Michelson Science Center at the California Institute of Technology.

\section{REFERENCES}

Akeson, R. L., Ciardi, D. R., van Belle, G. T., Creech-Eakman, M. J., \& Lada, E. A. 2000, ApJ, 543, 313

Akeson, R. L., et al. 2005a, ApJ, 635, 1173

. 2005b, ApJ, 622, 440

Baines, D., Oudmaijer, R. D., Porter, J. M., \& Pozzo, M. 2006, MNRAS, 367, 737

Baldwin, J. E., et al. 1996, A\&A, 306, L13

Berger, J.-P., et al. 2003, Proc. SPIE, 4838, 1099

Bergner, Y. K., Miroshnichenko, A. S., Yudin, R. V., Kuratov, K. S., Mukanov, D. B., \& Shejkina, T. A. 1995, A\&AS, 112, 221

Bertout, C., Robichon, N., \& Arenou, F. 1999, A\&A, 352, 574

Blake, G. A., \& Boogert, A. C. A. 2004, ApJ, 606, L73

Boden, A. F., et al. 2005, ApJ, 635, 442

Brittain, S. D., Rettig, T. W., Simon, T., Kulesa, C., DiSanti, M. A., \& Dello Russo, N. 2003, ApJ, 588, 535

Chen, W. P., Howell, R. R., Simon, M., \& Benson, J. A. 1992, ApJ, 387, L43

Chiang, E. I., \& Goldreich, P. 1997, ApJ, 490, 368

Cohen, M., \& Kuhi, L. V. 1979, ApJS, 41, 743

Cornwell, T. J., \& Wilkinson, P. N. 1981, MNRAS, 196, 1067

Corporon, P. 1998, Ph.D. thesis, Univ. Grenoble

Coudé du Foresto, V., Ridgway, S., \& Mariotti, J.-M. 1997, A\&AS, 121, 379

Coyne, G. V., \& Vrba, F. J. 1976, ApJ, 207, 790

D’Alessio, P., Canto, J., Calvet, N., \& Lizano, S. 1998, ApJ, 500, 411

de Winter, D., \& van den Ancker, M. E. 1997, A\&AS, 121, 275

Drew, J. E., Busfield, G., Hoare, M. G., Murdoch, K. A., Nixon, C. A., \& Oudmaijer, R. D. 1997, MNRAS, 286, 538

Duchêne, G., McCabe, C., Ghez, A. M., \& Macintosh, B. A. 2004, ApJ, 606, 969

Dullemond, C. P., Dominik, C., \& Natta, A. 2001, ApJ, 560, 957

Eisner, J. A., Lane, B. F., Akeson, R. L., Hillenbrand, L. A., \& Sargent, A. I. 2003, ApJ, 588, 360

Eisner, J. A., Lane, B. F., Hillenbrand, L. A., Akeson, R. L., \& Sargent, A. I. 2004, ApJ, 613, 1049

Elia, D., Strafella, F., Campeggio, L., Giannini, T., Lorenzetti, D., Nisini, B., \& Pezzuto, S. 2004, ApJ, 601, 1000

Finkenzeller, U. 1985, A\&A, 151, 340 
Grankin, K. N., et al. 1992, Inf. Bull. Variable Stars, 3747, 1

Haniff, C. A., Mackay, C. D., Titterington, D. J., Sivia, D., \& Baldwin, J. E. 1987, Nature, 328, 694

Harries, T. J., Monnier, J. D., Symington, N. H., \& Kurosawa, R. 2004, MNRAS, 350,565

Herbig, G. H., \& Bell, K. R. 1988, Catalog of Emission Line Stars of the Orion Population (Santa Cruz: Lick Obs.)

Herbst, W., Warner, J. W., Miller, D. P., \& Herzog, A. 1982, AJ, 87, 98

Hernández, J., Calvet, N., Briceño, C., Hartmann, L., \& Berlind, P. 2004, AJ, 127,1682

Hillenbrand, L. A., Strom, S. E., Vrba, F. J., \& Keene, J. 1992, ApJ, 397, 613

Høg, E., et al. 2000, A\&A, 355, L27

Hummel, C. A., Armstrong, J. T., Quirrenbach, A., Buscher, D. F., Mozurkewich, D., Elias, N. M., \& Wilson, R. E. 1994, AJ, 107, 1859

Hummel, C. A., Mozurkewich, D., Armstrong, J. T., Hajian, A. R., Elias, N. M., \& Hutter, D. J. 1998, AJ, 116, 2536

Ireland, M. J., Tuthill, P. G., Davis, J., \& Tango, W. 2005, MNRAS, 361, 337

Isella, A., \& Natta, A. 2005, A\&A, 438, 899

Jennison, R. C. 1958, MNRAS, 118, 276

Kharchenko, N. V. 2001, Kinem. Fiz. Nebesnykh Tel, 17, 409

Kraus, S., et al. 2005, AJ, 130, 246

Kurucz, R. L. 1979, ApJS, 40, 1

Lachaume, R. 2003, A\&A, 400, 795

Lachaume, R., Malbet, F., \& Monin, J.-L. 2003, A\&A, 400, 185

Leinert, C., Haas, M., Ábrahám, P., \& Richichi, A. 2001, A\&A, 375, 927

Leinert, C., Haas, M., Mundt, R., Richichi, A., \& Zinnecker, H. 1991, A\&A, 250,407

Leinert, C., Zinnecker, H., Weitzel, N., Christou, J., Ridgway, S. T., Jameson, R., Haas, M., \& Lenzen, R. 1993, A\&A, 278, 129

Li, W., Evans, N. J., Harvey, P. M., \& Colome, C. 1994, ApJ, 433, 199

Malbet, F., \& Bertout, C. 1995, A\&AS, 113, 369

Malbet, F., Lachaume, R., \& Monin, J.-L. 2001, A\&A, 379, 515

Malfait, K., Bogaert, E., \& Waelkens, C. 1998, A\&A, 331, 211

Millan-Gabet, R., Malbet, F., Akeson, R., Leinert, C., M. J. D., \& Water, R. 2006a, in Protostars and Planets V, ed. B. Reipurth et al. (Tucson: Univ. Arizona Press), in press

Millan-Gabet, R., \& Monnier, J. D. 2002, ApJ, 580, L167

Millan-Gabet, R., Schloerb, F. P., \& Traub, W. A. 2001, ApJ, 546, 358

Millan-Gabet, R., Schloerb, F. P., Traub, W. A., Malbet, F., Berger, J. P., \& Bregman, J. D. 1999, ApJ, 513, L131

Millan-Gabet, R., et al. 2006b, ApJ, 641, 547

. 2006c, ApJ, 645, L77

Miroshnichenko, A., Ivezić, Ž., Vinković, D., \& Elitzur, M. 1999a, ApJ, 520, L115

Miroshnichenko, A. S., Bjorkman, K. S., Grosso, M., Hinkle, K., Levato, H., \& Marang, F. 2005, A\&A, 436, 653

Miroshnichenko, A. S., Klochkova, V. G., Bjorkman, K. S., \& Panchuk, V. E. 2002, A\&A, 390, 627

Miroshnichenko, A. S., Mulliss, C. L., Bjorkman, K. S., Morrison, N. D., Glagolevskij, Y. V., \& Chountonov, G. A. 1998, PASP, 110, 883

Miroshnichenko, A. S., Mulliss, C. L., Bjorkman, K. S., Morrison, N. D., Kuratov, K. S., \& Wisniewski, J. P. 1999b, MNRAS, 302, 612
Monet, D. G., et al. 2003, AJ, 125, 984

Monnier, J. D. 2000, in Principles of Long Baseline Stellar Interferometry, ed.

P. R. Lawson (Pasadena: JPL), 203 2001, PASP, 113, 639

2003, Rep. Prog. Phys., 66, 789

Monnier, J. D., \& Millan-Gabet, R. 2002, ApJ, 579, 694

Monnier, J. D., et al. 2005, ApJ, 624, 832 2004, ApJ, 602, L57

Mora, A., et al. 2001, A\&A, 378, 116

Muzerolle, J., Calvet, N., Hartmann, L., \& D’Alessio, P. 2003, ApJ, 597, L149

Najita, J., Carr, J. S., \& Mathieu, R. D. 2003, ApJ, 589, 931

Natta, A., Prusti, T., Neri, R., Wooden, D., Grinin, V. P., \& Mannings, V. 2001, A\&A, 371, 186

Oudmaijer, R. D., \& Drew, J. E. 1999, MNRAS, 305, 166

Pauls, T. A., Young, J. S., Cotton, W. D., \& Monnier, J. D. 2005, PASP, 117, 1255

Pedretti, E., et al. 2004, PASP, 116, 377 2005, Appl. Opt., 44, 5173

Pérez, M. R., van den Ancker, M. E., de Winter, D., \& Bopp, B. W. 2004, A\&A, 416, 647

Perrin, G. 2003, A\&A, 398, 385

Pogodin, M. A., et al. 2004, A\&A, 417, 715

Pourbaix, D., \& Jorissen, A. 2000, A\&AS, 145, 161

Rettig, T., Brittain, S., Gibb, E., Balsara, D., \& Simon, T. 2005, BAAS, 207, 911

Skrutskie, M. F., Meyer, M. R., Whalen, D., \& Hamilton, C. 1996, AJ, 112, 2168

Stassun, K. G., Mathieu, R. D., Vaz, L. P. R., Stroud, N., \& Vrba, F. J. 2004, ApJS, 151, 357

Sylvester, R. J., Skinner, C. J., Barlow, M. J., \& Mannings, V. 1996, MNRAS, 279, 915

Tannirkulam, A., Monnier, J. D., \& Harries, T. J. 2005, BAAS, 207, 906

Thé, P. S., de Winter, D., \& Perez, M. R. 1994, A\&AS, 104, 315

Thompson, A. R., Moran, J. M., \& Swenson, G. W., Jr. 2001, Interferometry and Synthesis in Radio Astronomy (2nd ed.; New York: Wiley)

Traub, W. A., et al. 2003, Proc. SPIE, 4838, 45

Tuthill, P. G., Monnier, J. D., \& Danchi, W. C. 2001, Nature, 409, 1012

van Belle, G. T. 1999, PASP, 111, 1515

van Boekel, R., Dullemond, C. P., \& Dominik, C. 2005, A\&A, 441, 563

van Boekel, R., et al. 2004, Nature, 432, 479

van den Ancker, M. E., de Winter, D., \& Tjin A Djie, H. R. E. 1998, A\&A, 330,145

Vink, J. S., Drew, J. E., Harries, T. J., Oudmaijer, R. D., \& Unruh, Y. 2005, MNRAS, 359, 1049

Vinković, D., Ivezić, Ž., Jurkić, T., \& Elitzur, M. 2006, ApJ, 636, 348

Whitney, B. A., Wood, K., Bjorkman, J. E., \& Cohen, M. 2003, ApJ, 598, 1079

Wichmann, R., Bastian, U., Krautter, J., Jankovics, I., \& Rucinski, S. M. 1998, MNRAS, 301, L39

Wirnitzer, B. 1985, J. Opt. Soc. Am., 2, 14

Yoshida, S., Kogure, T., Nakano, M., Tatematsu, K., \& Wiramihardja, S. D. 1992, PASJ, 44, 77 